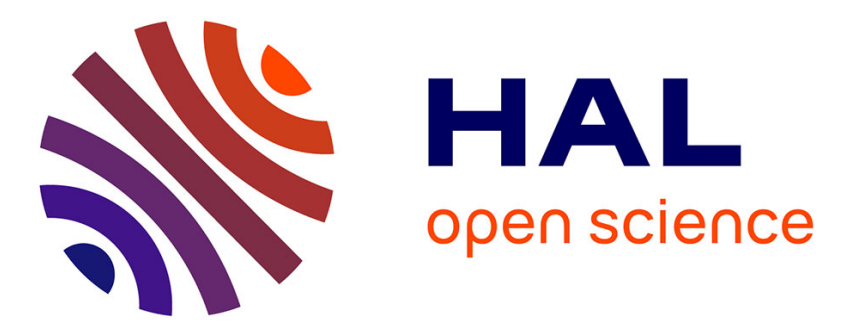

\title{
A computational strategy for thermo-poroelastic structures with a time-space interface coupling
}

David Néron, David Dureisseix

\section{To cite this version:}

David Néron, David Dureisseix. A computational strategy for thermo-poroelastic structures with a time-space interface coupling. International Journal for Numerical Methods in Engineering, 2007, 75 (9), pp.1053-1084. 10.1002/nme.2283 . hal-00325946

\section{HAL Id: hal-00325946 https://hal.science/hal-00325946}

Submitted on 1 Nov 2016

HAL is a multi-disciplinary open access archive for the deposit and dissemination of scientific research documents, whether they are published or not. The documents may come from teaching and research institutions in France or abroad, or from public or private research centers.
L'archive ouverte pluridisciplinaire HAL, est destinée au dépôt et à la diffusion de documents scientifiques de niveau recherche, publiés ou non, émanant des établissements d'enseignement et de recherche français ou étrangers, des laboratoires publics ou privés. 


\title{
A computational strategy for thermo-poroelastic structures with a time-space interface coupling
}

\author{
D. Néron ${ }^{1}$ and D. Dureisseix ${ }^{2}$
}

${ }^{1}$ LMT-Cachan (ENS de Cachan / CNRS UMR 8535 / Paris 6 University) 61, avenue du Président Wilson, F-94235 CACHAN CEDEX, FRANCE

${ }^{2}$ LMGC (Montpellier 2 University / CNRS UMR 5508 CC 048, place Eugène Bataillon, F-34095 MONTPELLIER CEDEX 5, FRANCE

\begin{abstract}
This paper deals with a computational strategy suitable for the simulation of multiphysics problems, based on the Large Time INcrement (LATIN) method. The simulation of such problems must encounter the possible different time and space scales which usually arise with the different physics. Herein, we focus on using different time and space discretizations for each physics by introducing an interface with its own discretization. The feasibility of both a time and space couplings is exemplified on a nonlinear 3-physics strongly coupled problem: the thermal / fluid / structure interaction in a thermo-poroelastic structure.

This is the post-print accepted version of the following article: D. Néron, D. Dureisseix, A computational strategy for thermo-poroelastic structures with a time-space interface coupling, International Journal for Numerical Methods in Engineering 75(9):1053-1084, Wiley, 2008, DOI: 10.1002/nme.2283, which has been published in final form at http://onlinelibrary.wiley.com/doi/10.1002/nme.2283/abstract
\end{abstract}

Keywords: multiphysics; thermo-poroelasticity; partitioning; LATIN; multiscale; finite elements; non matching meshes

\section{INTRODUCTION}

\subsection{Simulation of coupled multiphysics problems}

This paper deals with a computational strategy suitable for the simulation of multiphysics problems, based on the Large Time INcrement (LATIN) method [1]. The feasibility of this approach was presented in $[2,3,4]$ in the case of a strongly coupled problem: the consolidation of a saturated poroelastic structure in which the two physics involved were the fluid phase in the connected porosity and the solid phase as the skeleton of the porous medium.

A previous article [5] focused on the coupling of time discretizations when performing the simulation of this kind of 2-physics problem. The simplicity and the unidimensionality of the test case treated in this article allowed to derive an exact solution, to make some characteristics times appear and to eliminate the spatial aspect in order to focus the presentation on the time coupling aspect. The article led to the definition of time transfer operators between the different grids, as well as indications on the optimal choice of discretization for each physics (when comparing their own characteristic times). The criterion for the relative refinements in time was to obtain an iso-contribution to the global error of each physics. The conclusion was that the time step length ratio must be of the same magnitude that the ratio between the characteristic times of the physics involved. The use of an interface between physics with its own discretization was also discussed, showing the advantages in terms of modularity and cost reduction.

The comparison of this class of computational strategy with standard approaches can be found in [2].

\subsection{Motivation to use different space meshes as well}

The purpose of this first example is to exhibit the interest of using different spatial discretizations for the different physics by examining the results that are obtained when identical refined discretizations 
are used in order to compute a reference solution. In this context, simulations are performed using the monolithic approach, which consists in solving all the physics simultaneously. This method was presented in $[6,7,8]$ and was also recalled in $[2]$ for the case of porous media.

The proposed example concerns a pressurized filter made of porous ceramics whose characteristics have been chosen from PALL Schumacher Aerolith ${ }^{\circledR}$ material and will be given in Table 2. Such a device is typically used for filtration. Figure 1 shows the square cross section of the cylindrical filter and the pressure $p_{d}$ of the inflow fluid in the round bores. This pressure $p_{d}$ leads to boundary conditions on the porous medium: a prescribed pore pressure $p_{d}$ for the fluid on inner bores, and a prescribed force $\underline{F}_{d}=-p_{d} \underline{n}$ for the solid on the same part of the boundary. $p_{1}=36 \mathrm{MPa}$ at the time $t_{1}=0.345 \mathrm{~s}$. The remaining outflow boundary of the filter is assumed to be free in terms of displacement and submitted to the atmospheric pressure, for which the relative pressure is zero. The initial condition for the pore pressure is $p(t=0)=0$. The problem is modeled with the assumption of $2 \mathrm{D}$ plane strain and the symmetries are used to limit the study to a quarter of the section.

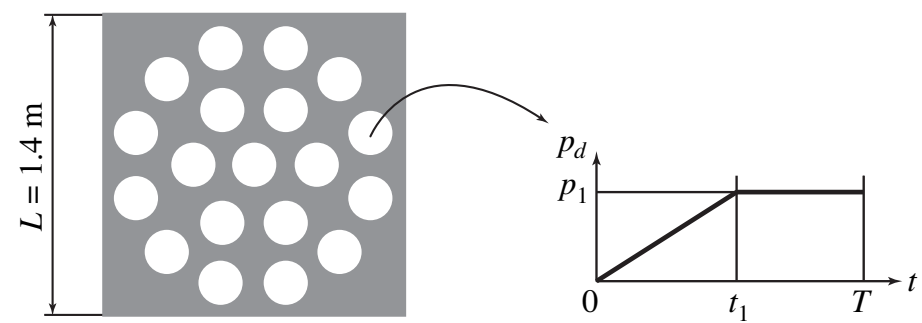

Figure 1: Section of the filter and pressure of the filtered fluid

Figure 2 shows the fields of the maximum principal strain and of the pore pressure in the domain at the final time step $T=0.69 \mathrm{~s}$. It is clear that high gradient zones are located between the bores for the strain and in the upper-right corner of the studied domain for the pore pressure. This statement motivates the idea of using different meshes for the solid and the fluid parts of the medium.

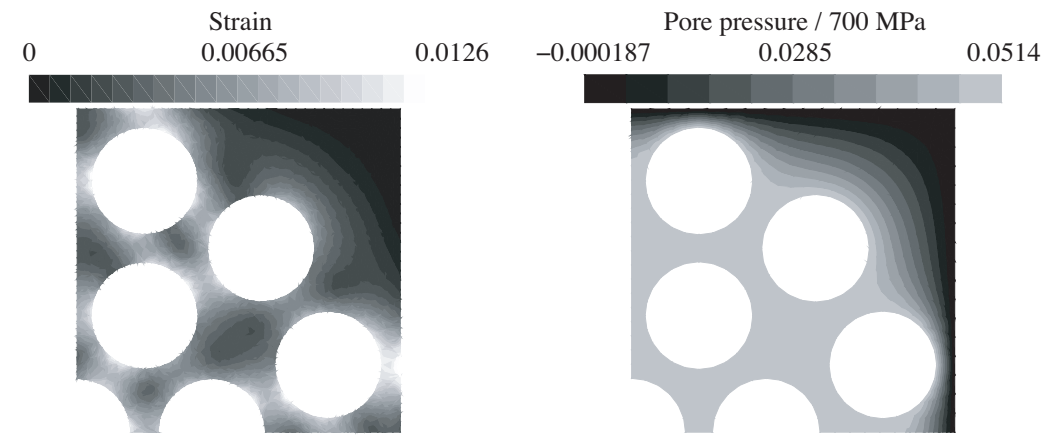

Figure 2: Maximum principal strain and pore pressure at the final time step

In the following, we are concerned in a problem where both the time and the space evolutions are discretized independently, and where three physics are strongly coupled: the thermal / fluid / structure interaction in a saturated porous medium. One can note that unlike other couplings such as aeroelasticity for which the problem is often loosely coupled, see $[9,10]$ for instance, this problem belongs to the strongly coupled category $[11,12]$.

Section 2 recalls the nonlinear thermo-poroelastic model that will serve as an illustration of the design of the computational strategy. The tools that allow to manage different time and space discretizations are developed in Section 3. Section 4 discusses the benefits and the drawbacks of using a direct monolithic approach or a partitioned strategy to deal with the different discretizations. Section 5 describes the solving procedure itself. Numerical issues and examples are proposed in the last Section. 


\section{THE THERMO-POROELASTIC MODEL FOR A SATU- RATED POROUS MEDIUM}

This Section selects as an illustrating case a nonlinear 3-physics coupled problem: the thermo-poroelastic quasi-static evolution on a time interval $[0, T]$ of a saturated porous medium $\Omega$. When the temperature is $T_{0}+\theta$, with small variations from reference absolute temperature $T_{0}$, and with small perturbations, the state of the material is described at each point of the structure with the following parameters:

- for the solid part (the ' $S$-physics'): the strain $\varepsilon$ associated to the stress $\boldsymbol{\sigma}$;

- for the fluid part (the ' $F$-physics'): the pore pressure $p$ associated to the fluid accumulation $v$ in the representative volume element;

- for the thermal part (the ' $T$-physics'): the temperature $\theta$ associated to the entropy $S$.

The loading on the medium consists of:

- prescribed body forces on the domain $\Omega$ (which will be assumed to be null for sake of simplicity in the presentation), prescribed displacement $\underline{U}_{d}$ on a first part $\partial_{1} \Omega$ of the boundary $\partial \Omega$ and traction force $\underline{F}_{d}$ on the complementary part $\partial_{2} \Omega$ of $\partial \Omega$;

- prescribed pore pressure $p_{d}$ on another part $\partial_{3} \Omega$ of the boundary and fluid flux $w_{d}$ on the complementary part $\partial_{4} \Omega$ of $\partial \Omega$;

- prescribed thermal source on the domain $\Omega$ (which will be assumed to be null for sake of simplicity in the presentation), prescribed temperature $\theta_{d}$ on another part $\partial_{5} \Omega$ of the boundary and, finally, thermal flux $G_{d}$ on the complementary part $\partial_{6} \Omega$ of $\partial \Omega$.

\subsection{Constitutive relations}

With the formalism of thermodynamics (for an internal variable description of the local behavior of the material) the thermo-poroelastic model considered herein is given by $[13,14]$ :

- the free energy $\Psi(\varepsilon, p, \theta)=\frac{1}{2} \operatorname{Tr}[\varepsilon \mathbf{D} \varepsilon]-b p \operatorname{Tr} \varepsilon-3 \alpha K_{b} \theta \operatorname{Tr} \varepsilon+3 \alpha_{m} p \theta-\frac{1}{2} \frac{1}{Q} p^{2}-\frac{1}{2} \frac{C_{d}}{T_{0}} \theta^{2}$;

- the dissipation potential $\Phi(\underline{Z}, \underline{X})=\frac{1}{2} \underline{Z} \cdot H \underline{Z}+\frac{1}{2} \frac{k}{T_{0}} \underline{X}^{2}$.

The coefficients of the material behavior model are the following: $\mathbf{D}$ is the Hooke operator of the drained skeleton; for an isotropic behavior, it depends only on two coefficients, for instance, Young modulus $E$ and Poisson coefficient $\nu . K_{b}=\frac{1}{3} E /(1-2 \nu)$ is the bulk modulus of the drained skeleton. $b$ is Biot coefficient: $b=1-K_{b} / K_{s}$, where $K_{s}$ is the bulk modulus of the solid phase (solid grains). $k$ is the thermal conductivity; as a first approximation, it can be chosen as $k=(1-n) k_{S}+n k_{F}, n$ being the porosity, $k_{S}$ and $k_{F}$ the thermal conductivities of the solid and fluid phases. $C_{d}$ is the heat capacity of the porous media; it is related to the specific heats of the phases, $c_{S}$ and $c_{F}$, by $C_{d}=\rho_{S} c_{S}+n \rho_{F} c_{F}$, $\rho_{S}$ and $\rho_{F}$ being the specific masses of the skeleton and fluid phases. $Q$ is Biot modulus; it takes into account the compressibility, $Q=\left((b-n) / K_{b}+n / K_{F}\right)^{-1}$, where $K_{F}$ is the compressibility of the fluid phase. $H=K / \mu_{F}$ is the permeability of the porous media; $K$ is the intrinsic permeability of the skeleton, and $\mu_{F}$ is the dynamic viscosity of the fluid phase. $\alpha$ is the thermal (lineic) expansion coefficient of the skeleton. Finally, $\alpha_{m}=n \alpha_{F}+(1-n) \alpha$ is the equivalent thermal expansion coefficient of the porous medium.

The state laws are therefore:

$$
\begin{gathered}
\boldsymbol{\sigma}=\frac{\partial \Psi}{\partial \varepsilon}=\mathbf{D} \boldsymbol{\varepsilon}-b p \mathbf{I}-3 \alpha K_{b} \theta \mathbf{I} \\
S=-\frac{\partial \Psi}{\partial \theta}=\frac{C_{d}}{T_{0}} \theta+3 \alpha K_{b} \operatorname{Tr} \varepsilon-3 \alpha_{m} p \\
v=-\frac{\partial \Psi}{\partial p}=\frac{1}{Q} p+b \operatorname{Tr} \varepsilon-3 \alpha_{m} \theta
\end{gathered}
$$


actually, we prefer to express the rate of fluid accumulation, $q=\dot{v}$, rather than the fluid accumulation itself, i.e.

$$
q=\frac{1}{Q} \dot{p}+b \operatorname{Tr} \dot{\varepsilon}-3 \alpha_{m} \dot{\theta}
$$

The gradients involved are: $\underline{Z}=\operatorname{grad} p$ the gradient of the pore pressure and $\underline{X}=\operatorname{grad} \theta$ the gradient of the macroscopic temperature. The evolution laws are:

$$
\begin{aligned}
\underline{Y} & =\frac{\partial \Phi}{\partial \underline{X}}=\frac{k}{T_{0}} \underline{X} \\
\underline{W} & =\frac{\partial \Phi}{\partial \underline{Z}}=H \underline{Z}
\end{aligned}
$$

where $\underline{W}$ denotes the opposite of Darcy velocity, and $\underline{Y}=-\frac{q_{\mathrm{th}}}{T_{0}}, \underline{q}_{\mathrm{th}}$ being the thermal flux.

Once the constitutive relations are settled for the material behavior, the conservation principles have to be established for the structure behavior: the momentum conservation for the solid, the mass conservation for the fluid, and the heat equation. These are the global admissibility relations. They are detailed in the following and some functional spaces are introduced. For any affine space $\square$, notation $\square_{0}$ denotes the associated vector space.

\section{$2.2 \quad S$-physics admissibility}

Concerning the solid problem, one must have a compatible strain field that derives from the symmetric part of the gradient of a displacement field $\underline{U}$. This field should be regular and equal the prescribed displacement $\underline{U}_{d}$ on a first part $\partial_{1} \Omega$ of the boundary $\partial \Omega$ of the domain: $\underline{U}_{\mid \partial_{1} \Omega}=\underline{U}_{d}$. The corresponding space of displacement fields $\underline{U}$ is denoted $\mathcal{U}$. Then, one should have:

$$
\varepsilon=(\operatorname{Grad} \underline{U})_{\mathrm{sym}} \text { and } \underline{U} \in \mathcal{U}
$$

The corresponding space of displacements and strain fields $(\underline{U}, \varepsilon)$ is denoted $\mathcal{E}$.

For the momentum conservation, the stress field should also balance the external prescribed forces $\underline{F}_{d}$ on the complementary part $\partial_{2} \Omega$ of the domain (for sake of simplicity, no body force is considered herein): $\boldsymbol{\sigma} \underline{n}_{\mid \partial_{2} \Omega}=\underline{F}_{d}$ and $\underline{\operatorname{div}} \boldsymbol{\sigma}=0$ in $\Omega$. The variational formulation is, at each time step:

$$
\forall\left(\underline{U}^{\star}, \varepsilon^{\star}\right) \in \mathcal{E}_{0}, \quad \int_{\Omega} \operatorname{Tr}\left[\sigma \dot{\varepsilon}^{\star}\right] d \Omega=\int_{\partial_{2} \Omega} \underline{F}_{d} \cdot \underline{\dot{U}}^{\star} d S
$$

The corresponding space of stress fields $\sigma$ is denoted $\mathcal{S}$.

\section{$2.3 \quad F$-physics admissibility}

Concerning the fluid problem, the pressure gradient should derive from a regular pore pressure field that equals the prescribed values $p_{d}$ on another part of the boundary $\partial_{3} \Omega: p_{\mid \partial_{3} \Omega}=p_{d}$. The corresponding space of pressure fields $p$ is denoted $\mathcal{P}$. Then, one should have:

$$
\underline{Z}=\underline{\operatorname{grad}} p \quad \text { and } \quad p \in \mathcal{P}
$$

The corresponding space of pressure and pressure gradient fields $(p, \underline{Z})$ is denoted $\mathcal{Z}$.

The fluid flux must equal the prescribed values on the complementary part $\partial_{4} \Omega$ of the boundary: $\underline{W} \cdot \underline{n}_{\mid \partial_{4} \Omega}=w_{d}$. The conservation of fluid flow on each elementary representative volume reads: $q=$ $\operatorname{div} \underline{W}$. The variational formulation is, at each time step:

$$
\forall\left(p^{\star}, \underline{Z}^{\star}\right) \in \mathcal{Z}_{0}, \quad \int_{\Omega}\left(q p^{\star}+\underline{W} \cdot \underline{Z}^{\star}\right) d \Omega=\int_{\partial_{4} \Omega} w_{d} p^{\star} d S
$$

The corresponding space of fluid accumulation rate and flux fields $(q, \underline{W})$ is denoted $\mathcal{W}$. 


\section{$2.4 \quad T$-physics admissibility}

Concerning the thermal problem, one must have a temperature gradient $\underline{X}$ arising from a regular temperature field $\theta$ that equals the prescribed value $\theta_{d}$ on another part $\partial_{5} \Omega$ of the boundary : $\theta_{\mid \partial_{5} \Omega}=\theta_{d}$. The corresponding space of temperature fields $\theta$ is denoted $\mathcal{T}$. Then, one should have:

$$
\underline{X}=\underline{\operatorname{grad}} \theta \text { and } \theta \in \mathcal{T}
$$

The corresponding space of temperature and temperature gradient fields $(\theta, \underline{X})$ is denoted $\mathcal{X}$.

The thermal flux must equal the prescribed values on the complementary part $\partial_{6} \Omega$ of the boundary: $\underline{q}_{\mathrm{th}} \cdot \underline{n}_{\mid \partial_{6} \Omega}=G_{d}$, therefore $\underline{Y} \cdot \underline{n}_{\mid \partial_{6} \Omega}=-G_{d} / T_{0}$. No convection is assumed herein. Finally, the energy balance of the first thermodynamic principle has to be settled. With no thermal source, and following [13] for instance, one gets the local thermal balance equation on $\Omega$ :

$$
R=\operatorname{div} \underline{Y}
$$

with

$$
R=\frac{C_{d}}{T_{0}} \dot{\theta}+3 \alpha K_{b} \operatorname{Tr} \dot{\varepsilon}-3 \alpha_{m} \dot{p}-\frac{\rho_{F} c_{F}}{T_{0}} \underline{W} \cdot \underline{X}-\frac{1}{H}\left(\frac{1}{T_{0}}-3 \alpha_{F}\right) \underline{W^{2}}
$$

The last two nonlinear terms correspond to the heat convectively transported by the fluid, and to the viscous dissipation. Depending on the application, they can sometimes be neglected. The variational formulation is, at each time step:

$$
\forall\left(\theta^{\star}, \underline{X}^{\star}\right) \in \mathcal{X}_{0}, \quad \int_{\Omega}\left(R \theta^{\star}+\underline{Y} \cdot \underline{X}^{\star}\right) d \Omega=\int_{\partial_{6} \Omega}-\frac{G_{d}}{T_{0}} \theta^{\star} d S
$$

The corresponding space of fields $(R, \underline{Y})$ is denoted $\mathcal{Y}$.

\section{DEALING WITH DIFFERENT DISCRETIZATIONS}

The case of the coupling of time discretizations has been handled in [5]. In the following, an identical framework is used for defining space transfer operators. However, the context is slightly different because of the points where the quantities considered are defined.

Indeed, when using a finite element discretization, some of the fields are defined at the nodes of the mesh (e.g. the displacement), while, due to numerical integration considerations, some others are defined at the integration points (e.g. the stress and the strain) [15]. Especially for the visualization of the solution, these fields can be extrapolated from the integration points to the nodes of the mesh, in order to be interpolated using the finite element shape functions; but the extrapolated fields are not necessary continuous throughout the element edges.

In the strategy that will be presented in Section 5, we are concerned with the transfer of thermoporoelasticity space fields (e.g. the stress $\sigma$ for the $S$-physics or the fluid accumulation $q$ for the $F$-physics) which are only computed at the integration points. This characteristic implies a slight modification of the technique which was introduced in [5].

It is to be noted that, unlike previous works on space multiscale approach in the context of the LATIN method $[16,17,18]$; the element sizes of the 3 meshes we intend to use are not clearly separated: the proposed approach should be able to deal with a mesh for the $S$-physics which is refined in certain areas while the mesh for the $F$-physics can be refined in other ones; therefore, no discretization is embedded into any other.

\subsection{Space transfer of discretized fields}

Consider the case of the transfer between non matching finite element meshes $\Omega_{1}$ and $\Omega_{2}$. A first application of the following technique was described and tested in [19] for thermo-viscoelasticity and only the basic ideas will be recalled herein.

The procedure that is described in [19] starts by an extrapolation of the fields and it is the discontinuity of the extrapolated fields which requires a special attention during the transfer. The overall procedure consists in several steps:

- the sampled field given at integration points of the first mesh $\Omega_{1}$ is extrapolated (at the element level) to the nodes of the same mesh; 
- this new field is transferred to the second mesh $\Omega_{2}$ (the transferred field does not require a continuity condition);

- this field is finally interpolated (at the element level) to the integration points of the last mesh $\Omega_{2}$.

In the following, the procedure will be illustrated in the case of the fluid accumulation space field $q_{1}$, defined on $\Omega_{1}$, transferred to $q_{2}$, defined on $\Omega_{2}$.

Basically, one needs an extrapolation of $Q_{1}$, the column vector of values of the fluid accumulation at integration points of mesh $\Omega_{1}$, to the nodes of the element within which the integration points are located. A least square minimization allows to extrapolate the values at nodes of the element, stored in the column vector $\mathcal{Q}_{1}$. This field is easily interpolated within each element using $\boldsymbol{N}_{1}$, the finite element shape functions basis restricted to each element of the mesh $\Omega_{1}: q_{1}(M)=\boldsymbol{N}_{1}(M) \mathcal{Q}_{1} . q_{1}$ is not continuous throughout the element edges; this does not cause any problem since the considered fields do not require $\mathcal{C}^{0}$ regularity, therefore the various jumps between elements do not involve any energy.

We are now concerned with the transfer of $q_{1}(M)$, defined on $\Omega_{1}$, to $q_{2}(M)$, defined on $\Omega_{2}$, and we choose to express the conservation of the 'energy' scalar product with respect to a set of test functions $p_{2}^{\star}(M)$ as:

$$
\int_{\Omega_{2}} q_{2} p_{2}^{\star} d \Omega_{2}=\int_{\Omega_{1}} q_{1} p_{2}^{\star} d \Omega_{1}
$$

which can be interpreted as the conservation of generalized averages of $q_{1}$ with respect to the test functions. The proposed test function basis is $N_{2}$, the finite element shape functions restricted to each element of the target mesh (Figure 3).

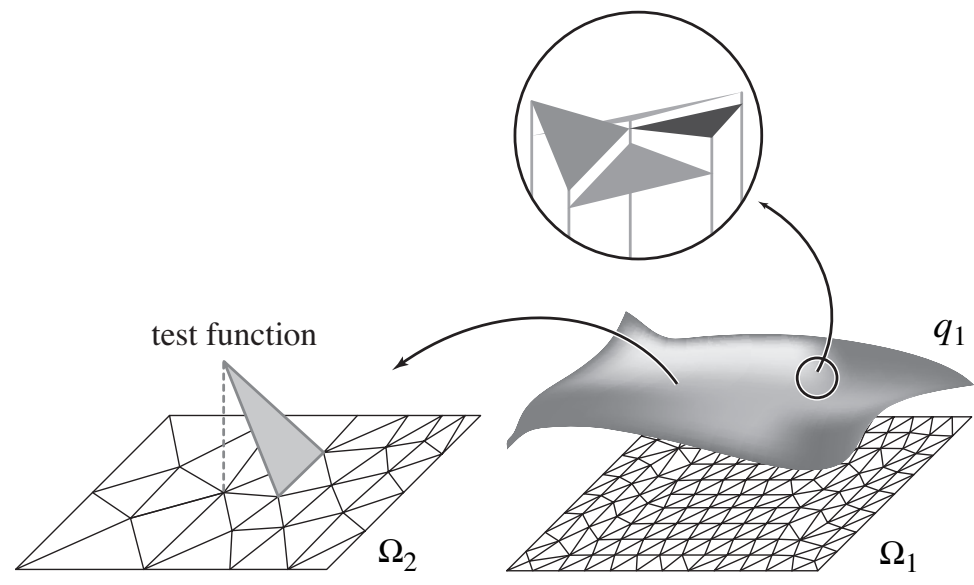

Figure 3: A proposed space discontinuous test function

In such a way, the previously extrapolated field $q_{1}=N_{1} \mathcal{Q}_{1}$ is projected to a field $q_{2}=N_{2} \mathcal{Q}_{2}$, also discontinuous throughout element edges, such that:

$$
\mathcal{Q}_{2}=\mathcal{G}_{21} \mathcal{Q}_{1} \quad \text { with } \quad \mathcal{G}_{21}=\boldsymbol{M}_{2}^{-1} \boldsymbol{M}_{21}
$$

$\boldsymbol{M}_{2}$ is the 'mass' matrix of shape functions $\boldsymbol{N}_{2}$ of $\Omega_{2}$. It collects as blocks on the diagonal the 'mass' matrices of the target elements $\Omega_{2}^{e}$ :

$$
\boldsymbol{M}_{2}^{e}=\int_{\Omega_{2}^{e}} \boldsymbol{N}_{2}^{T} \boldsymbol{N}_{2} d \Omega
$$

Applying the projection requires the use of $\left(\boldsymbol{M}_{2}^{e}\right)^{-1}$, but, as it is related to each element independently, $\boldsymbol{M}_{2}^{e}$ is small-sized and therefore the overall projection is not CPU expensive. $\boldsymbol{M}_{21}$ is the matrix of cross product of shape functions: it can be computed on the intersection of source and target elements $\Omega_{1}^{e}$ and $\Omega_{2}^{e}$ only:

$$
\boldsymbol{M}_{21}=\int_{\Omega_{1}} \boldsymbol{N}_{1}^{T} \boldsymbol{N}_{2} d \Omega
$$

To avoid a CPU overhead, only neighboring elements of $\Omega_{1}$ and $\Omega_{2}$ (with a non void intersection) are selected once for all with a 2-level search. Intersection of elements is performed using classical tools of Computational Geometry [20] and numerical integration of $\boldsymbol{M}_{21}$ is performed accordingly. All the required operations during a space field projection are performed element by element. 
The resulting field $q_{2}$ is finally interpolated on each target element with the shape functions $\boldsymbol{N}_{2}$ to obtain $Q_{2}$, the column vector of values of the fluid accumulation at integration points of mesh $\Omega_{2}$. All these operations allow to define the transfer operator $\boldsymbol{P}_{21}$ such that $Q_{2}=\boldsymbol{P}_{21} Q_{1}$ as well as the dual transfer operator $\boldsymbol{P}_{12}$ using in the same way $\mathcal{G}_{12}=\boldsymbol{M}_{1}^{-1} \boldsymbol{M}_{12}$.

Let us mention that these transfer operators are closely related to mortar projections [21, 22] and that with the previous choice of extrapolation, projection and interpolation, one can prove the following properties [19]:

- conservation of 'energy' scalar product: $\int_{\Omega_{2}} q_{2} p_{2} d \Omega_{2}=\int_{\Omega_{1}} q_{1} p_{1} d \Omega_{1}$, if $q_{2}=\boldsymbol{P}_{21} q_{1}$ and $p_{1}=\boldsymbol{P}_{12} p_{2}$;

- exact transfer of a field which can be represented both on $\Omega_{1}$ and $\Omega_{2}$.

The extension to operators that transfer tensors — such as the stress field $\boldsymbol{\sigma}$ - or vectors, is trivial by building them component by component of the field.

It has to be noted that the discretized geometries may be not exactly compatible and that the discretized boundaries do not match exactly. The dedicated treatment of non-matching geometries is detailed in [19], and re-used herein.

\subsection{Time transfer}

Due to different involved physics, with different characteristic times, each may possess its own time discretization and time transfer operators are also required. The previous space transfer operations lead to element by element computations based on discontinuous fields. To design the time transfer operators, we wish to reuse the same tools; therefore, we have to deal with a time discretization that allows jumps from a time slab to the other, and that provides a variational framework. As detailed in [5], the time discontinuous Galerkin (TDG) approach (see [23, 24]) is such a candidate; it is used in all of the following.

Briefly, one considers that a field $e(t)$ is represented on a time interval $[0, T]$, with linear (for degree one interpolation) evolution on each time slab $\left[t_{i}, t_{i+1}\right]$ (for $i=0 \ldots n, t_{0}=0, t_{n}=T$ ), but with possible jumps at time instants $t_{i}$. The representation of the evolution on each time step requires two values: $e_{i}^{+}=\lim _{t \rightarrow t_{i}^{+}} e(t)$ and $e_{i+1}^{-}=\lim _{t \rightarrow t_{i+1}^{-}} e(t)$, and the derivative $\dot{e}$ is not defined at these particular instants. Therefore, all of the operations involving such a quantity have to be expressed in a variational form, where the following integral is usually defined as:

$$
\int_{[0, T]} \dot{e} e^{\star} d t:=\sum_{i=0}^{n-1}\left\{\int_{] t_{i}, t_{i+1}[} \dot{e} e^{\star} d t+\left[e_{i}^{+}-e_{i}^{-}\right] e_{i}^{\star,+}\right\}
$$

for all fields $e^{\star}$ defined with the same time discontinuous approximation as for $e, e_{0}^{-}$being defined as the initial condition.

The corresponding time transfer operators (detailed in [5]) will be denoted in the following by $\boldsymbol{p}_{12}$ for a transfer operator from time discretization $T_{2}$ to $T_{1}$.

\subsection{Space-time transfer}

In this article, we focus on problems where both the space and time discretizations are different for each physic. The interesting feature is the 'associative property' of transfer mechanisms provided by time and space transfer operators: each can be applied at first or at last without changing the overall result. This is exemplified if the discretized values of a field $e_{1}(M, t)$ (defined on $T_{1} \times \Omega_{1}$ ) are stored in a matrix $\boldsymbol{E}_{1}$ with the total number of integration points as rows, and the number of time steps as columns; in this case, one gets for the matrix of the transferred field $e_{2}(M, t)\left(\right.$ defined on $\left.T_{2} \times \Omega_{2}\right)$ :

$$
\boldsymbol{E}_{2}=\boldsymbol{P}_{21} \boldsymbol{E}_{1} \boldsymbol{p}_{21}^{T}
$$

This operation will be formally denoted with $\boldsymbol{E}_{2}=\mathcal{P}_{21} \boldsymbol{E}_{1}$, or even abusively $e_{2}=\mathcal{P}_{12} e_{1}$, in the following.

\section{MONOLITHIC VS. PARTITIONING APPROACHES}

When dealing with identical discretizations for each physics, an illustration of the monolithic approach for the case of a thermo-hydro-mechanical process can be found in [25]. To illustrate the advantages of a 
partitioning strategy if one wishes to use different discretizations, we derive herein a tentative description of a monolithic approach. The resulting formulation is then discussed to emphasize its drawbacks.

\subsection{Monolithic approach coupling two spatial discretizations for a poroelastic model}

In order to simplify the presentation, we select a 2-physics version of the previous problem, by decoupling the thermal evolution from the poroelastic one: the coupling coefficients $\alpha$ and $\alpha_{m}$ are chosen equal to zero. We are therefore interested in the poroelastic part of the problem only. In the following monolithic method, only the case of different space discretizations is considered.

After space discretization, the coupled constitutive relations (1a),(2),(3b) are rewritten using the transfer operators, and are restricted to the solid and fluid quantities:

$$
\begin{gathered}
\sigma_{S}=D \varepsilon_{S}-b B_{e}^{T} \boldsymbol{P}_{S F} P_{F} \\
W_{F}=H Z_{F} \quad \text { and } \quad Q_{F}=\frac{1}{Q} \dot{P}_{F}+b \boldsymbol{P}_{F S} B_{e} \dot{\varepsilon}_{S}
\end{gathered}
$$

where all quantities are the values at integration points of $\Omega_{S}$ when subscript ' $S$ ' is used and of $\Omega_{F}$ when subscript ' $F$ ' is used. These equations are a priori non local in space, due to the transfer operators. $B_{e}$ is such that $\operatorname{Tr} \varepsilon_{S}=B_{e} \varepsilon_{S}$.

In the same way, $S$-admissibility conditions $(4),(5)$ are discretized:

$$
\begin{aligned}
\varepsilon_{S} & =B_{\varepsilon} U_{S} \\
\forall U_{S}^{\star} \in \mathcal{U}_{0}, \quad U_{S}^{\star T} B_{\sigma}^{T} \sigma_{S} & =U_{S}^{\star T} f_{d S}
\end{aligned}
$$

where $U_{S}$ is the displacement field at the nodes of mesh $\Omega_{S} ; \varepsilon_{S}$ and $\sigma_{S}$ are the strain and stress fields at the integration points of mesh $\Omega_{S} . B_{\sigma}$ and $B_{\varepsilon}$ are classical finite element operators. $f_{d S}$ are the external generalized prescribed forces.

Fluid admissibility conditions (6),(7) are discretized:

$$
\begin{aligned}
Z_{F} & =B_{z} p_{F} \\
\forall p_{F}^{\star} \in \mathcal{P}_{0}, \quad p_{F}^{\star T}\left(B_{q}^{T} Q_{F}+B_{w}^{T} W_{F}\right) & =p_{F}^{\star T} g_{d F}
\end{aligned}
$$

where $p_{F}$ is the pore pressure field at the nodes of mesh $\Omega_{F} ; Z_{F}, Q_{F}$ and $W_{F}$ are fields defined at the integration points of mesh $\Omega_{F} . B_{q}, B_{w}$ and $B_{z}$ are finite element operators for the fluid discretization. $g_{d F}$ are the external generalized prescribed fluxes. Finally, one needs the operator $B_{p}$, such that $P_{F}=B_{p} p_{F}$ is the pore pressure field at integration points of $\Omega_{F}$, interpolated from the nodal pore pressure $p_{F}$.

If we use the discretized constitutive relations (18),(19) together with the discretized admissibility conditions (20) and (21), the 2-mesh monolithic problem is:

$$
\begin{array}{r}
U_{S}^{\star T}[\underbrace{\left(B_{\sigma}^{T} D B_{\varepsilon}\right)}_{K} U_{S}-\underbrace{\left(B_{\sigma}^{T} b B_{e}^{T} \boldsymbol{P}_{S F} B_{p}\right)}_{A_{S F}} p_{F}]=U_{S}^{\star T} f_{d S} \\
p_{F}^{\star T}[\underbrace{\left(B_{q}^{T} \frac{1}{Q} B_{p}\right)}_{S} \dot{p}_{F}+\underbrace{\left(B_{w}^{T} H B_{z}\right)}_{H} p_{F}+\underbrace{\left(B_{q}^{T} b \boldsymbol{P}_{F S} B_{e} B_{\varepsilon}\right)}_{A_{F S}} \dot{U}_{S}]=p_{F}^{\star T} g_{d F}
\end{array}
$$

Omitting the prescribed kinematic quantities to simplify the presentation, this leads to:

$$
\left[\begin{array}{cc}
0 & 0 \\
A_{F S} & S
\end{array}\right]\left[\begin{array}{c}
\dot{U}_{S} \\
\dot{p}_{F}
\end{array}\right]+\left[\begin{array}{cc}
K & -A_{S F} \\
0 & H
\end{array}\right]\left[\begin{array}{l}
U_{S} \\
p_{F}
\end{array}\right]=\left[\begin{array}{l}
f_{d S} \\
g_{d F}
\end{array}\right]
$$

or, with a time derivative of the first group of equations:

$$
\left[\begin{array}{cc}
K & -A_{S F} \\
-A_{F S} & -S
\end{array}\right]\left[\begin{array}{c}
\dot{U}_{S} \\
\dot{p}_{F}
\end{array}\right]+\left[\begin{array}{cc}
0 & 0 \\
0 & -H
\end{array}\right]\left[\begin{array}{c}
U_{S} \\
p_{F}
\end{array}\right]=\left[\begin{array}{c}
\dot{f}_{d S} \\
-g_{d F}
\end{array}\right]
$$

With the chosen transfer operators, this problem can be proved to be symmetric. Nevertheless, its resolution is very expensive due to the high fill in of the involved submatrices $A_{F S}$ and $A_{S F}$. Moreover, this kind of approach requires the development of a specific code for each multiphysics problem. 
One can notice that if identical meshes are used, transfer operators are equal to identity matrices and one recovers the classical monolithic approach described in [2].

If one considers also different time discretizations, the ordinary differential system (24) is no longer local in time variable, and is even more costly. For the use of an incremental monolithic scheme with different time discretizations, an approach can be found in [26]. Different time discretizations of the same physical evolution, in an iterative manner that allows resolutions in parallel (though with a somehow poor parallel efficiency) can be found in [27, 28].

\subsection{Partitioning approaches}

Partitioning approaches, which basically consist in separating the physics in order to avoid the simultaneous treatment of the different fields, are usually preferred to the direct monolithic analysis because they offer several interesting features including:

- the ability to use different discretizations for each physics;

- the simplification in software development efforts;

- preservation of software modularity.

These advantages to solve coupled multiphysics problems have been mentioned in a broad range of cases, such as fluid/structure interactions in $[29,30,31,32,33,14,34]$ among others. In particular, the issue of the data exchange between two different physics has been studied with different approaches in $[33,34]$ and the allowance of different treatments (different softwares, integration schemes...) for the different physics involved has been highlighted in [33, 35, 36, 37, 38, 39, 40].

The LATIN approach that will be used in the following is one of these partitioning approaches. As presented in [5], using an interface between the physics with its own discretization, increases the previous modularity in the solution procedure, as well as in the modeling of the coupled phenomena. For instance, if a physical model changes, or if a third physics is added, a corresponding time-space domain can be added, while the new couplings enrich the interface behavior (see Figure 4). It also leads to savings in the CPU overhead, especially with both non-matching time and space discretizations for which the transfer of space-time fields from one discretization to the other may be costly.
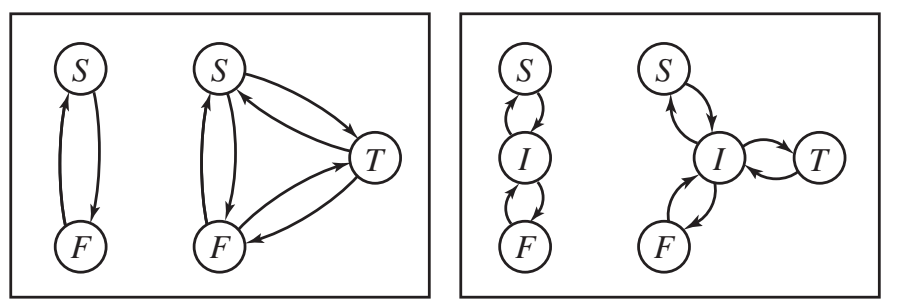

Figure 4: Modifications of transfer operations when adding a new physics (left: without interface, right: with a dedicated interface discretization)

\section{THE LATIN APPROACH WITH AN INTERFACE BETWEEN PHYSICS}

The Large Time INcrement method (LATIN) is a general mechanics-based computational strategy originally designed for solving time-dependent nonlinear problems [1]. The extension of this strategy for multiphysics problems has been reported in $[2,3,41,5]$. Its main originality lies in a non-incremental iterative approach which operates over the entire time-space domain.

\subsection{The Large Time INcrement method for multiphysics problems}

The solution that has to be found is denoted with $\mathbf{s}=\left(\mathbf{s}_{S}, \mathbf{s}_{F}, \mathbf{s}_{T}\right)$ where $\mathbf{s}_{S}=(\varepsilon, \boldsymbol{\sigma}), \mathbf{s}_{F}=(p, q, \underline{Z}, \underline{W})$ and $\mathbf{s}_{T}=(\theta, R, \underline{X}, \underline{Y})$. The 10 fields involved are defined on the whole time-space domain $[0, T] \times \Omega$ and have to verify the following equations:

- the admissibility of the $S$-physics (4),(5); 
- the admissibility of the $F$-physics (6),(7);

- the admissibility of the $T$-physics (8),(9);

- the behavior of the interface coupling all the physics.

The first three conditions are global-in-space 'monophysics' problems which can be solved using specific codes over possibly different time-space domains $T_{S} \times \Omega_{S}, T_{F} \times \Omega_{F}$ and $T_{T} \times \Omega_{T}$. The last condition is a 'multiphysics' problem. However, it is local in the space variable and, thus, it can be solved relatively easily, even if it is nonlinear. It can be interpreted as the behavior of a particular medium, the interface between physics, and we summarize it with:

$$
\begin{aligned}
\boldsymbol{\sigma} & =\mathbf{D} \boldsymbol{\varepsilon}-b p \mathbf{I}-3 \alpha K_{b} \theta \mathbf{I} \\
q & =\frac{1}{Q} \dot{p}+b \operatorname{Tr} \dot{\boldsymbol{\varepsilon}}-3 \alpha_{m} \dot{\theta} \\
\underline{W} & =H \underline{Z} \\
\underline{Y} & =\frac{k}{T_{0}} \underline{X} \\
R & =\frac{C_{d}}{T_{0}} \dot{\theta}+3 \alpha K_{b} \operatorname{Tr} \dot{\boldsymbol{\varepsilon}}-3 \alpha_{m} \dot{p}-\frac{\rho_{F} c_{F}}{T_{0}} \underline{W} \cdot \underline{X}-\frac{1}{H}\left(\frac{1}{T_{0}}-3 \alpha_{F}\right) \underline{W^{2}}
\end{aligned}
$$

Since all these equations are defined on the same interface, all are solved over the same time domain $T_{I}$, and independently at each point of the space domain $\Omega_{I}$.

For such a problem, the LATIN method is built with three principles:

- the first principle consists in splitting the difficulties to avoid the simultaneous treatment of two difficulties: globality of the equations to solve and coupling between physics. The first set of equations, $\mathbf{A}_{\mathbf{d}}$, contains the global but uncoupled field admissibilities of the $S, F$ and the $T$-physics $(4),(5),(6),(7),(8),(9)$. The second set, $\boldsymbol{\Gamma}$, contains the local and coupled behavior of the interface between physics (25), together with initial conditions. Thus, the solution $\mathbf{s}_{e x}$ of the problem is the intersection of $\mathbf{A}_{\mathbf{d}}$ and $\boldsymbol{\Gamma}$, i.e. $\mathbf{s}_{e x}=\mathbf{A}_{\mathbf{d}} \cap \boldsymbol{\Gamma}$;

- the second principle consists in using an iterative procedure, producing alternatively elements of $\boldsymbol{\Gamma}$ and $\mathbf{A}_{\mathbf{d}}$, up to the convergence. Iteration $n+1$ contains two stages: (i) once a solution $\mathbf{s}_{n} \in \mathbf{A}_{\mathbf{d}}$ is known, the local stage consists in finding a solution $\hat{\mathbf{s}}_{n+1 / 2} \in \boldsymbol{\Gamma}$, using a first search direction $\mathbf{E}^{+}$, (ii) once a solution $\hat{\mathbf{s}}_{n+1 / 2} \in \boldsymbol{\Gamma}$ is known, the decoupled stage consists in finding a solution $\mathbf{s}_{n+1} \in \mathbf{A}_{\mathbf{d}}$, using a second search direction $\mathbf{E}^{-}$. The particular expressions of these search directions will be precised in the following Sections. If they are constant along iterations, and conjugate with each other, a proof of convergence of the algorithm can be built, following [1].

- the third principle consists in taking into account the fact that the algorithm provides solutions at each iteration that are defined on the whole domain and the whole time interval, to use a suited representation on the unknowns, at least on $\mathbf{A}_{\mathbf{d}}$. This step is crucial to gain efficiency of the method. For poroelastic problems, a partial representation of the sole kinematic quantities was used in [2]. As in [3], but where only mono-dimensional poroelastic problems where treated, we use herein the representation of all quantities of $\mathbf{A}_{\mathbf{d}}$ in order to decrease the storage requirements of the algorithm. This technics will be described in the next Sections.

Figure 5 attempts to offer a 'geometrical' interpretation of the method, figuring the various sets of equations $\mathbf{A}_{\mathbf{d}}, \boldsymbol{\Gamma}, \mathbf{E}^{+}$and $\mathbf{E}^{-}$in the space generated by $(\varepsilon, p, \underline{Z}, \theta, \underline{X})$ and $(\boldsymbol{\sigma}, q, \underline{W}, R, \underline{Y})$.

\subsection{Local stage at Iteration $n+1$}

Suppose $\mathbf{s}_{n}=\left(\mathbf{s}_{S}, \mathbf{s}_{F}, \mathbf{s}_{T}\right)$ is a known element of $\mathbf{A}_{\mathbf{d}}$, where $\mathbf{s}_{S}=(\varepsilon, \boldsymbol{\sigma}), \mathbf{s}_{F}=(p, q, \underline{Z}, \underline{W})$ and $\mathbf{s}_{T}=$ $(\theta, R, \underline{X}, \underline{Y})$ are defined on their time-space domains $T_{S} \times \Omega_{S}, T_{F} \times \Omega_{F}$ and $T_{T} \times \Omega_{T}$ (which are possibly different). The local stage consists in finding $\hat{\mathbf{s}}_{n+1 / 2}=(\hat{\varepsilon}, \hat{\boldsymbol{\sigma}}, \hat{p}, \hat{q}, \underline{\hat{Z}}, \underline{\hat{W}}, \hat{\theta}, \hat{R}, \underline{\hat{X}}, \underline{\hat{Y}})$ in $\boldsymbol{\Gamma}$, i.e. satisfying the constitutive relations (25) at each time step and each point of the time-space domain $T_{I} \times \Omega_{I}$ (the 


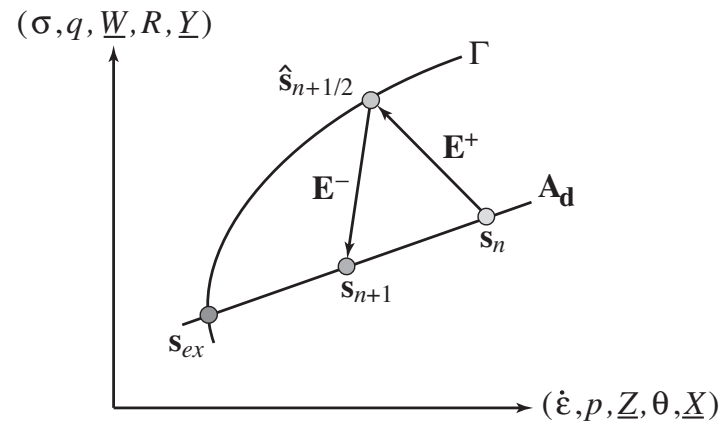

Figure 5: Iteration $n+1$ of the LATIN method

interface between the physics). To close the problem, one has to add the search direction $\mathbf{E}^{+}$. Following [2], it can be chosen of the form:

$$
\begin{aligned}
(\hat{\boldsymbol{\sigma}}-\boldsymbol{\sigma})+(\mathbf{L} \hat{\dot{\varepsilon}}-\mathbf{L} \dot{\boldsymbol{\varepsilon}}) & =0 \\
(\hat{q}-q)+(r \hat{p}-r p) & =0 \\
(\underline{\hat{W}}-\underline{W})+(H \underline{\hat{Z}}-H \underline{Z}) & =0 \\
(\hat{R}-R)+(u \hat{\theta}-u \theta) & =0 \\
(\underline{\hat{Y}}-\underline{Y})+\left(\frac{k}{T_{0}} \underline{\hat{X}}-\frac{k}{T_{0}} \underline{X}\right) & =0
\end{aligned}
$$

where $\mathbf{L}, r$ and $u$ are the parameters of the method which do not influence the solution once convergence has been reached.

If the time evolution of all the quantities that are involved in the problem are chosen in the representation space, denoted $\mathcal{T}_{I}$, of TDG functions on the interface time discretization $T_{I}$, the search direction $\mathbf{E}^{+}$has to be expressed with a time variational form and the equations are not strictly local in time (they are nevertheless local to each time slab). Choosing the test functions in the same space recovers the time locality only for the previous equations that does not involve time derivative, but the first search direction equation must be rewritten in the form:

$$
\forall \boldsymbol{\sigma}^{\star} \in \mathcal{T}_{I}, \int_{T_{I}} \operatorname{Tr}\left[((\hat{\boldsymbol{\sigma}}-\boldsymbol{\sigma})+(\mathbf{L} \hat{\dot{\varepsilon}}-\mathbf{L} \dot{\boldsymbol{\varepsilon}})) \mathbf{L}^{-1} \boldsymbol{\sigma}^{\star}\right] d t=0
$$

which must be understood as:

$$
\begin{aligned}
& \forall \boldsymbol{\sigma}^{\star} \in \mathcal{T}_{I} \\
& \int_{T_{I}} \operatorname{Tr}\left[(\hat{\boldsymbol{\sigma}}-\boldsymbol{\sigma}) \mathbf{L}^{-1} \boldsymbol{\sigma}^{\star}\right] d t+\sum_{i=0}^{n-1}\left\{\int_{]_{i}, t_{i+1}[} \operatorname{Tr}\left[(\hat{\hat{\varepsilon}}-\dot{\boldsymbol{\varepsilon}}) \boldsymbol{\sigma}^{\star}\right] d t+\operatorname{Tr}\left[\left((\hat{\boldsymbol{\varepsilon}}-\boldsymbol{\varepsilon})_{i}^{+}-(\hat{\boldsymbol{\varepsilon}}-\boldsymbol{\varepsilon})_{i}^{-}\right) \boldsymbol{\sigma}_{i}^{\star,+}\right]\right\}=0
\end{aligned}
$$

and the initial conditions lead to $(\hat{\varepsilon}-\varepsilon)_{0}^{-}=\hat{\varepsilon}_{0}^{-}-\varepsilon_{0}^{-}=0$.

For sake of simplicity in this presentation, the time variational form will be used for all the equations, even if some of them can be rewritten locally. For those for which it is not possible, such as the first search direction equation, the previous definition will be used.

The second modification of the search direction is due to the fact that all the involved quantities are not defined on the same discretization grids. Since both time and space discretizations could be different, space-time transfer operators have to be used. Actually, three transfer operators are required since quantities defined on $S, F$, and $T$-physics have to be transferred onto the interface: $\mathcal{P}_{I S}, \mathcal{P}_{I F}$ and $\mathcal{P}_{I T}$ are time-space transfer operators that transfer to $T_{I} \times \Omega_{I}$ a field defined on the time-space domain $T_{S} \times \Omega_{S}$, respectively $T_{F} \times \Omega_{F}$ and $T_{T} \times \Omega_{T}$. Finally, using $S, F, T$ and $I$ subscripts to recall the 
time-space discretization onto the various quantities are defined, the search direction now reads:

$$
\begin{array}{r}
\forall \boldsymbol{\sigma}_{I}^{\star} \in \mathcal{T}_{I}, \int_{T_{I}} \operatorname{Tr}\left[\left(\left(\hat{\boldsymbol{\sigma}}_{I}-\mathcal{P}_{I S} \boldsymbol{\sigma}_{S}\right)+\frac{d}{d t}\left(\mathbf{L} \hat{\boldsymbol{\varepsilon}}_{I}-\mathcal{P}_{I S} \mathbf{L} \boldsymbol{\varepsilon}_{S}\right)\right) \mathbf{L}^{-1} \boldsymbol{\sigma}_{I}^{\star}\right] d t=0 \\
\forall q_{I}^{\star} \in \mathcal{T}_{I}, \int_{T_{I}}\left(\left(\hat{q}_{I}-\mathcal{P}_{I F} q_{F}\right)+\left(r \hat{p}_{I}-\mathcal{P}_{I F} r p_{F}\right)\right) r^{-1} q_{I}^{\star} d t=0 \\
\forall \underline{W}_{I}^{\star} \in \mathcal{T}_{I}, \int_{T_{I}}\left(\left(\underline{\hat{W}}_{I}-\mathcal{P}_{I F} \underline{W}_{F}\right)+\left(H \underline{\hat{Z}}_{I}-\mathcal{P}_{I F} H \underline{Z}_{F}\right)\right) \cdot H^{-1} \underline{W}_{I}^{\star} d t=0 \\
\forall R_{I}^{\star} \in \mathcal{T}_{I}, \int_{T_{I}}\left(\left(\hat{R}_{I}-\mathcal{P}_{I T} R_{T}\right)+\left(u \hat{\theta}_{I}-\mathcal{P}_{I T} u \theta_{T}\right)\right) u^{-1} R_{I}^{\star} d t=0 \\
\forall \underline{Y}_{I}^{\star} \in \mathcal{T}_{I}, \int_{T_{I}}\left(\left(\underline{\hat{Y}}_{I}-\mathcal{P}_{I T} \underline{Y}_{T}\right)+\left(\frac{k}{T_{0}} \underline{\hat{X}}_{I}-\mathcal{P}_{I T} \frac{k}{T_{0}} \underline{X}_{T}\right)\right) \cdot\left(\frac{k}{T_{0}}\right)^{-1} \underline{Y}_{I}^{\star} d t=0
\end{array}
$$

where $\frac{d_{I}}{d t}$ denotes the derivation on the time discretization $T_{I}$. Note that a monoscale method similar to the one in [2] is recovered as soon as transfer operators are identity operators.

Expressing the constitutive relations (25) in the same TDG framework, the previous search direction lead to an explicit solution for:

$$
\begin{gathered}
\underline{\hat{W}}_{I}=H \underline{\hat{Z}}_{I}=\frac{1}{2} \mathcal{P}_{I F}\left(\underline{W}_{F}+H \underline{Z}_{F}\right) \\
\underline{\hat{Y}}_{I}=\frac{k}{T_{0}} \underline{\hat{X}}_{I}=\frac{1}{2} \mathcal{P}_{I T}\left(\underline{Y}_{T}+\frac{k}{T_{0}} \underline{X}_{T}\right)
\end{gathered}
$$

and once $\underline{\hat{W}}_{I}$ and $\underline{X}_{I}$ are known, they can be considered as a given right hand side in the remaining quasi-local and small-sized coupled first order differential system:

$$
\begin{array}{r}
\forall \boldsymbol{\sigma}_{I}^{\star} \in \mathcal{T}_{I}, \int_{T_{I}} \operatorname{Tr}\left[\left(\mathbf{L} \frac{d_{I}}{d t} \hat{\boldsymbol{\varepsilon}}_{I}+\mathbf{D} \hat{\varepsilon}_{I}-b \hat{p}_{I} \mathbf{I}-3 \alpha K_{b} \hat{\theta}_{I} \mathbf{I}-\mathbf{A}_{I}\right) \mathbf{L}^{-1} \boldsymbol{\sigma}_{I}^{\star}\right] d t=0 \\
\forall q_{I}^{\star} \in \mathcal{T}_{I}, \int_{T_{I}}\left(r \hat{p}_{I}+\frac{d_{I}}{d t}\left(\frac{1}{Q} \hat{p}_{I}+b \operatorname{Tr} \hat{\varepsilon}_{I}-3 \alpha_{m} \hat{\theta}_{I}\right)-\alpha_{I}\right) r^{-1} q_{I}^{\star} d t=0 \\
\forall R_{I}^{\star} \in \mathcal{T}_{I}, \int_{T_{I}}\left(u \hat{\theta}_{I}+\frac{d_{I}}{d t}\left(\frac{C_{d}}{T_{0}} \hat{\theta}_{I}+3 \alpha K_{b} \operatorname{Tr} \hat{\varepsilon}_{I}-3 \alpha_{m} \hat{p}_{I}\right)-\gamma_{I}\right) u^{-1} R_{I}^{\star} d t=0
\end{array}
$$

with the known quantities at this stage:

$$
\begin{aligned}
\mathbf{A}_{I} & =\mathcal{P}_{I S} \boldsymbol{\sigma}_{S}+\frac{d_{I}}{d t}\left(\mathcal{P}_{I S} \mathbf{L} \varepsilon_{S}\right) \\
\alpha_{I} & =\mathcal{P}_{I F}\left(q_{F}+r p_{F}\right) \\
\gamma_{I} & =\frac{\rho_{F} c_{F}}{T_{0}} \underline{\hat{W}}_{I} \cdot \underline{\hat{X}}_{I}+\frac{1}{H}\left(\frac{1}{T_{0}}-3 \alpha_{F}\right) \underline{\hat{W}}_{I}^{2}+\mathcal{P}_{I T}\left(R_{T}+u \theta_{T}\right)
\end{aligned}
$$

and for which the initial conditions have to be added.

\subsection{Decoupled stage at Iteration $n+1$ with representation of the unknowns}

This stage, for poroelastic problems, has been previously described in [3], where no transfer operator was used. It is briefly recalled herein with such transfer operators, and for the thermo-poroelastic case.

Suppose $\hat{\mathbf{s}}_{n+1 / 2}=(\hat{\varepsilon}, \hat{\boldsymbol{\sigma}}, \hat{p}, \hat{q}, \underline{\hat{Z}}, \underline{\hat{W}}, \hat{\theta}, \hat{R}, \underline{\hat{X}}, \underline{\hat{Y}})$ is a known element of $\boldsymbol{\Gamma}$, with all the fields being defined on $T_{I} \times \Omega_{I}$. The decoupled stage consists in finding $\mathbf{s}_{n+1}=\left(\mathbf{s}_{S}, \mathbf{s}_{F}, \mathbf{s}_{T}\right)$ in $\mathbf{A}_{\mathbf{d}}$, where $\mathbf{s}_{S}=(\varepsilon, \boldsymbol{\sigma})$, $\mathbf{s}_{F}=(p, q, \underline{Z}, \underline{W})$ and $\mathbf{s}_{T}=(\theta, R, \underline{X}, \underline{Y})$ are respectively defined on $T_{S} \times \Omega_{S}, T_{F} \times \Omega_{F}$ and $T_{T} \times \Omega_{T}$, and verify the decoupled admissibility conditions (4), (5), (6), (7), (8) and (9). To close the problem, one has to add the second search direction $\mathbf{E}^{-}$, conjugate to the previous one, so that the $S, F$ and the 
$T$-physics problems remain decoupled:

$$
\begin{array}{r}
\forall \boldsymbol{\sigma}_{S}^{\star} \in \mathcal{T}_{S}, \int_{T_{S}} \operatorname{Tr}\left[\left(\left(\boldsymbol{\sigma}_{S}-\mathcal{P}_{S I} \hat{\boldsymbol{\sigma}}_{I}\right)-\frac{d_{S}}{d t}\left(\mathbf{L} \boldsymbol{\varepsilon}_{S}-\mathcal{P}_{S I} \mathbf{L} \hat{\varepsilon}_{I}\right)\right) \mathbf{L}^{-1} \boldsymbol{\sigma}_{S}^{\star}\right] d t=0 \\
\forall q_{F}^{\star} \in \mathcal{T}_{F}, \int_{T_{F}}\left(\left(q_{F}-\mathcal{P}_{F I} \hat{q}_{I}\right)-\left(r p_{F}-\mathcal{P}_{F I} r \hat{p}_{I}\right)\right) r^{-1} q_{F}^{\star} d t=0 \\
\forall \underline{W}_{F}^{\star} \in \mathcal{T}_{F}, \int_{T_{F}}\left(\left(\underline{W}_{F}-\mathcal{P}_{F I} \underline{W}_{I}\right)-\left(H \underline{Z}_{F}-\mathcal{P}_{F I} H \underline{Z}_{I}\right)\right) \cdot H^{-1} \underline{W}_{F}^{\star} d t=0 \\
\forall R_{T}^{\star} \in \mathcal{T}_{T}, \int_{T_{T}}\left(\left(R_{T}-\mathcal{P}_{T I} \hat{R}_{I}\right)-\left(u \hat{\theta}_{T}-\mathcal{P}_{T I} u \hat{\theta}_{I}\right)\right) u^{-1} R_{T}^{\star} d t=0 \\
\forall \underline{Y}_{T}^{\star} \in \mathcal{T}_{T}, \int_{T_{T}}\left(\left(\underline{Y}_{T}-\mathcal{P}_{T I} \underline{\hat{Y}}_{I}\right)-\left(\frac{k}{T_{0}} \underline{X}_{T}-\mathcal{P}_{T I} \frac{k}{T_{0}} \underline{X}_{I}\right)\right) \cdot\left(\frac{k}{T_{0}}\right)^{-1} \underline{Y}_{T}^{\star} d t=0
\end{array}
$$

where $\frac{d_{S}}{d t}$ denotes the derivation on time discretization $T_{S}$, and where $\mathcal{T}_{S}, \mathcal{T}_{F}$ and $\mathcal{T}_{I}$ are the representation spaces of TDG functions on time discretizations $T_{S}, T_{F}, T_{T}$. Furthermore, with the third principle of the LATIN method, we search $\mathbf{s}_{S}, \mathbf{s}_{F}$ and $\mathbf{s}_{T}$ in an adaptive fashion, representing them as a sum of products of space fields by scalar time functions. This technique, which is commonly used in the LATIN method [1], is denoted as 'radial loading approximation'. Usually, at iteration $n+1$, one new couple of space field and scalar time function is added for each field to those already available at the previous iteration $n$, i.e., formally:

- for the solid quantities, $\mathbf{s}_{S}^{(n+1)}=\mathbf{s}_{S}^{(n)}+\Delta \mathbf{s}_{S}$;

- for the fluid quantities, $\mathbf{s}_{F}^{(n+1)}=\mathbf{s}_{F}^{(n)}+\Delta \mathbf{s}_{F}$;

- for the thermal quantities, $\mathbf{s}_{T}^{(n+1)}=\mathbf{s}_{T}^{(n)}+\Delta \mathbf{s}_{T}$

where each field corresponding to corrections $\Delta \mathbf{s}_{S}, \Delta \mathbf{s}_{F}$ and $\Delta \mathbf{s}_{T}$ is represented using the radial loading approximation. Search direction (33) is rewritten in terms of corrections:

$$
\begin{array}{r}
\forall \boldsymbol{\sigma}_{S}^{\star} \in \mathcal{T}_{S}, \int_{T_{S}} \operatorname{Tr}\left[\left(\Delta \boldsymbol{\sigma}_{S}-\frac{d_{S}}{d t} \mathbf{L} \Delta \boldsymbol{\varepsilon}_{S}+\hat{\mathbf{A}}_{S}\right) \mathbf{L}^{-1} \boldsymbol{\sigma}_{S}^{\star}\right] d t=0 \\
\forall q_{F}^{\star} \in \mathcal{T}_{F}, \int_{T_{F}}\left(\Delta q_{F}-r \Delta p_{F}+\hat{\alpha}_{F}\right) r^{-1} q_{F}^{\star} d t=0 \\
\forall \underline{W}_{F}^{\star} \in \mathcal{T}_{F}, \int_{T_{F}}\left(\Delta \underline{W}_{F}-H \Delta \underline{Z}_{F}+\underline{\beta}_{F}\right) \cdot H^{-1} \underline{W}_{F}^{\star} d t=0 \\
\forall R_{T}^{\star} \in \mathcal{T}_{T}, \int_{T_{T}}\left(\Delta R_{T}-u \Delta \hat{\theta}_{T}+\hat{\gamma}_{T}\right) u^{-1} R_{T}^{\star} d t=0 \\
\forall \underline{Y}_{T}^{\star} \in \mathcal{T}_{T}, \int_{T_{T}}\left(\Delta \underline{Y}_{T}-\frac{k}{T_{0}} \Delta \underline{X}_{T}+\underline{\hat{\delta}}_{T}\right) \cdot\left(\frac{k}{T_{0}}\right)^{-1} \underline{Y}_{T}^{\star} d t=0
\end{array}
$$

with the known quantities at this stage:

$$
\begin{aligned}
\hat{\mathbf{A}}_{S} & =\left(\boldsymbol{\sigma}_{S}-\frac{d_{S}}{d t} \mathbf{L} \boldsymbol{\varepsilon}_{S}\right)-\left(\mathcal{P}_{S I} \hat{\boldsymbol{\sigma}}_{I}-\frac{d}{d t} \boldsymbol{\mathcal { P }}_{S I} \mathbf{L} \boldsymbol{\varepsilon}_{I}\right) \\
\hat{\alpha}_{F} & =\left(q_{F}-r p_{F}\right)-\boldsymbol{\mathcal { P }}_{F I}\left(\hat{q}_{I}-r \hat{p}_{I}\right) \\
\hat{\hat{\beta}}_{F} & =\left(\underline{W}_{F}-H \underline{Z}_{F}\right)-\mathcal{P}_{F I}\left(\underline{\hat{W}}_{I}-H \underline{\hat{Z}}_{I}\right) \\
\hat{\gamma}_{T} & =\left(R_{T}-u \theta_{T}\right)-\boldsymbol{\mathcal { P }}_{T I}\left(\hat{R}_{I}-u \hat{\theta}_{I}\right) \\
\hat{\hat{\delta}}_{T} & =\left(\underline{Y}_{T}-\frac{k}{T_{0}} \underline{X}_{T}\right)-\mathcal{P}_{T I}\left(\underline{\hat{Y}}_{I}-\frac{k}{T_{0}} \underline{\hat{X}}_{I}\right)
\end{aligned}
$$

As $\mathbf{s}_{S}, \mathbf{s}_{F}$ and $\mathbf{s}_{T}$ must satisfy the admissibility equations both at iteration $n$ and at iteration $n+1$, corrections $\Delta \mathbf{s}_{S}, \Delta \mathbf{s}_{F}$ and $\Delta \mathbf{s}_{T}$ must also satisfy these equations but for homogenous conditions. The way to build these corrections is described in the following Subsections. 


\subsubsection{S-physics}

Correction $\Delta \mathbf{s}_{S}=\left(\Delta \varepsilon_{S}, \Delta \boldsymbol{\sigma}_{S}\right)$ is defined on $T_{S} \times \Omega_{S}$ and has to satisfy the admissibility conditions (4), (5) for homogenous conditions, i.e. $\Delta \mathbf{s}_{S} \in \mathcal{E}_{0} \times \mathcal{S}_{0}$. This reads, using the TDG scheme:

$$
\begin{array}{r}
\forall \boldsymbol{\sigma}_{S}^{\star} \in \mathcal{S}_{0}, \quad \int_{T_{S} \times \Omega_{S}} \operatorname{Tr}\left[\boldsymbol{\sigma}_{S}^{\star} \frac{d_{S}}{d t} \Delta \varepsilon_{S}\right] d \Omega d t=0 \\
\forall \underline{U}_{S}^{\star} \in \mathcal{U}_{0}, \quad \int_{T_{S} \times \Omega_{S}} \operatorname{Tr}\left[\Delta \boldsymbol{\sigma}_{S} \frac{d_{S}}{d t} \varepsilon\left(\underline{U}_{S}^{\star}\right)\right] d \Omega d t=0
\end{array}
$$

Using equation (34a) of the search direction together with the admissibility condition (36) leads to find $\Delta \sigma_{S} \in \mathcal{S}_{0}$ such that:

$$
\forall \boldsymbol{\sigma}_{S}^{\star} \in \mathcal{S}_{0}, \quad \int_{T_{S} \times \Omega_{S}} \operatorname{Tr}\left[\left(\Delta \boldsymbol{\sigma}_{S}+\hat{\mathbf{A}}_{S}\right) \mathbf{L}^{-1} \boldsymbol{\sigma}_{S}^{\star}\right] d \Omega d t=0
$$

The unknown field $\Delta \boldsymbol{\sigma}_{S}$ is approximated by:

$$
\Delta \boldsymbol{\sigma}_{S}(M, t)=\boldsymbol{S}_{S}(M) s_{S}(t)
$$

where $s_{S}$ is a scalar time function belonging to $\mathcal{T}_{S}$, while $\boldsymbol{S}_{S}$ is a space field which belongs to $\mathcal{S}_{0}$. Test function $\boldsymbol{\sigma}_{S}^{\star}$ is chosen as $\boldsymbol{\sigma}_{S}^{\star}=\boldsymbol{S}_{S} s_{S}^{\star}+\boldsymbol{S}_{S}^{\star} s_{S}$, with $\boldsymbol{S}_{S}^{\star} \in \mathcal{S}_{0}$ and $s_{S}^{\star} \in \mathcal{T}_{S}$, such that (38) leads to the coupled system:

$$
\begin{aligned}
& \forall \boldsymbol{S}_{S}^{\star} \in \mathcal{S}_{0}, \quad \int_{T_{S} \times \Omega_{S}} \operatorname{Tr}\left[s_{S}\left(s_{S} \boldsymbol{S}_{S}+\hat{\mathbf{A}}_{S}\right) \mathbf{L}^{-1} \boldsymbol{S}_{S}^{\star}\right] d \Omega d t=0 \\
& \forall s_{S}^{\star} \in \mathcal{T}_{S}, \quad \int_{T_{S} \times \Omega_{S}} \operatorname{Tr}\left[s_{S}^{\star}\left(s_{S} \boldsymbol{S}_{S}+\hat{\mathbf{A}}_{S}\right) \mathbf{L}^{-1} \boldsymbol{S}_{S}\right] d \Omega d t=0
\end{aligned}
$$

Equation (40a) is dualized using a Lagrange multiplier $\underline{X}_{S} \in \mathcal{U}_{0}$ and the normalization:

$$
\int_{\Omega_{S}} \operatorname{Tr}\left[\boldsymbol{S}_{S} \mathbf{L}^{-1} \boldsymbol{S}_{S}\right] d \Omega=1
$$

is enforced to get a unique couple $\left(\boldsymbol{S}_{S}, s_{S}\right)$ as a solution. This leads to a coupled system of equations with $\left(\boldsymbol{S}_{S}, s_{S}\right)$ as unknowns:

$$
\begin{array}{r}
\forall s_{S}^{\star} \in \mathcal{T}_{S}, \quad \int_{T_{S}} s_{S}^{\star}\left(s_{S}+\int_{\Omega_{S}} \operatorname{Tr}\left[\hat{\mathbf{A}}_{S} \mathbf{L}^{-1} \boldsymbol{S}_{S}\right] d \Omega\right) d t=0 \\
\boldsymbol{S}_{S}=\frac{1}{\left\|s_{S}\right\|_{T_{S}}^{2}}\left(\mathbf{L} \varepsilon\left(\underline{X}_{S}\right)-\int_{T_{S}} s_{S} \hat{\mathbf{A}}_{S} d t\right) \quad \text { with } \underline{X}_{S} \in \mathcal{U}_{0} \text { such that } \\
\forall \underline{X}_{S}^{\star} \in \mathcal{U}_{0}, \quad \int_{\Omega_{S}} \operatorname{Tr}\left[\varepsilon\left(\underline{X}_{S}\right) \mathbf{L} \varepsilon\left(\underline{X}_{S}^{\star}\right)\right] d \Omega=\int_{\Omega_{S}} \operatorname{Tr}\left[\left(\int_{T_{S}} s_{S} \hat{\mathbf{A}}_{S} d t\right) \varepsilon\left(\underline{X}_{S}^{\star}\right)\right] d \Omega
\end{array}
$$

where $\left\|s_{S}\right\|_{T_{S}}^{2}=\int_{T_{S}} s_{S}^{2} d t$. System (42) is solved using a fixed-point method between (a) and (b). The number of subiterations is usually small (typically only 2 subiterations).

The unknown field $\Delta \varepsilon_{S}$ is approximated by:

$$
\Delta \varepsilon_{S}(M, t)=\varepsilon\left(\underline{V}_{S}(M)\right) v_{S}(t)
$$

where $v_{S}$ is scalar time function belonging $\mathcal{T}_{S}$, while $\underline{V}_{S}$ is a space field which belongs to $\mathcal{U}_{0}$.

One possibility, which is easy to implement, is to chose $\underline{V}_{S}=\underline{X}_{S}$ and then $\Delta \varepsilon_{S}=\varepsilon\left(\underline{X}_{S}\right) v_{S}$. In Equation (34a) of the search direction, test function $\boldsymbol{\sigma}_{S}^{\star}$ is chosen as $\boldsymbol{\sigma}_{S}^{\star}=\mathbf{L} \varepsilon\left(\underline{X}_{S}\right) v_{S}^{\star}$, with $s_{S}^{\star} \in \mathcal{T}_{S}$, which leads to the determination of $v_{S}$ by solving:

$$
\forall v_{S}^{\star} \in \mathcal{T}_{S}, \quad \int_{T_{S}} v_{S}^{\star} \int_{\Omega_{S}} \operatorname{Tr}\left[\left(\frac{d_{S}}{d t} v_{S} \mathbf{L} \varepsilon\left(\underline{X}_{S}\right)-\hat{\mathbf{A}}_{S}\right) \varepsilon\left(\underline{X}_{S}\right)\right] d \Omega d t
$$

i.e.:

$$
\forall v_{S}^{\star} \in \mathcal{T}_{S}, \quad \int_{\Omega_{S}} \operatorname{Tr}\left[\varepsilon\left(\underline{X}_{S}\right) \mathbf{L} \varepsilon\left(\underline{X}_{S}\right)\right] d \Omega \int_{T_{S}} v_{S}^{\star}\left(\frac{d_{S}}{d t} v_{S}\right) d t=\int_{T_{S}} v_{S}^{\star} \int_{\Omega_{S}} \operatorname{Tr}\left[\hat{\mathbf{A}}_{S} \varepsilon\left(\underline{X}_{S}\right)\right] d \Omega d t
$$




\subsubsection{F-physics}

Correction $\Delta \mathbf{s}_{F}=\left(\Delta p_{F}, \Delta \underline{Z}_{F}, \Delta q_{F}, \Delta \underline{W}_{F}\right)$ is defined on $T_{F} \times \Omega_{F}$ and has to satisfy the admissibility conditions (6), (7) for homogenous conditions, i.e. $\Delta \mathbf{s}_{F} \in \mathcal{Z}_{0} \times \mathcal{W}_{0}$. This reads, using the TDG scheme:

$$
\begin{aligned}
& \forall\left(q_{F}^{\star}, \underline{W}_{F}^{\star}\right) \in \mathcal{W}_{0}, \quad \int_{T_{F} \times \Omega_{F}}\left(q_{F}^{\star} \Delta p_{F}+\underline{W}_{F}^{\star} \cdot \Delta \underline{Z}_{F}\right) d \Omega d t=0 \\
& \forall\left(p_{F}^{\star}, \underline{Z}_{F}^{\star}\right) \in \mathcal{Z}_{0}, \quad \int_{T_{F} \times \Omega_{F}}\left(\Delta q_{F} p_{F}^{\star}+\Delta \underline{W}_{F} \cdot \underline{Z}_{F}^{\star}\right) d \Omega d t=0
\end{aligned}
$$

Using equations (34b), (34c) of the search direction together with the admissibility condition (46) leads to find $\left(\Delta q_{F}, \Delta \underline{W}_{F}\right) \in \mathcal{W}_{0}$ such that:

$$
\forall\left(q_{F}^{\star}, \underline{W}_{F}^{\star}\right) \in \mathcal{W}_{0}, \quad \int_{T_{F} \times \Omega_{F}}\left(\left(\Delta q_{F}+\hat{\alpha}_{F}\right) r^{-1} q_{F}^{\star}+\left(\Delta \underline{W}_{F}+\underline{\hat{\beta}}_{F}\right) \cdot H^{-1} \underline{W}_{F}^{\star}\right) d \Omega d t=0
$$

The unknown fields $\left(\Delta q_{F}, \Delta \underline{W}_{F}\right)$ are approximated by:

$$
\Delta q_{F}(M, t)=Q_{F}(M) \phi_{F}(t) \quad \text { and } \quad \Delta \underline{W}_{F}(M, t)=\underline{J}_{F}(M) \phi_{F}(t)
$$

where $\phi_{F}$ is a scalar time function belonging to $\mathcal{T}_{F}$, while $\left(Q_{F}, J_{F}\right)$ is a couple of space fields which belongs to $\mathcal{W}_{0}$. Test functions $q_{F}^{\star}$ and $\underline{W}_{F}^{\star}$ are chosen as $q_{F}^{\star}=Q_{F} \phi_{F}^{\star}+Q_{F}^{\star} \phi_{F}$ and $\underline{W}_{F}^{\star}=\underline{J}_{F} \phi_{F}^{\star}+\underline{J}_{F}^{\star} \phi_{F}$, with $\left(Q_{F}^{\star}, J_{F}^{\star}\right) \in \mathcal{W}_{0}$ and $\phi_{F}^{\star} \in \mathcal{T}_{F}$, such that (48) leads to the coupled system:

$$
\begin{aligned}
\forall\left(Q_{F}^{\star}, \underline{J}_{F}^{\star}\right) \in \mathcal{W}_{0}, \quad \int_{T_{F} \times \Omega_{F}} \phi_{F}\left(\left(\phi_{F} Q_{F}+\hat{\alpha}_{F}\right) r^{-1} Q_{F}^{\star}+\left(\phi_{F} \underline{Y}_{F}+\underline{\hat{\beta}}_{F}\right) \cdot H^{-1} \underline{J}_{F}^{\star}\right) d \Omega d t=0 \\
\forall \phi_{F}^{\star} \mathcal{T}_{F}, \quad \int_{T_{F} \times \Omega_{F}} \phi_{F}^{\star}\left(\left(\phi_{F} Q_{F}+\hat{\alpha}_{F}\right) r^{-1} Q_{F}+\left(\phi_{F} \underline{Y}_{F}+\underline{\hat{\beta}}_{F}\right) \cdot H^{-1} \underline{J}_{F}\right) d \Omega d t=0
\end{aligned}
$$

Equation (50a) is dualized using a couple of Lagrange multipliers $\left(\Pi_{F}, \Xi_{F}\right) \in \mathcal{Z}_{0}$ and the normalization:

$$
\int_{\Omega_{F}}\left(Q_{F} r^{-1} Q_{F}+\underline{J}_{F} \cdot H^{-1} \underline{J}_{F}\right) d \Omega=1
$$

is enforced to get a unique triplet $\left(Q_{F}, \underline{J}_{F}, \phi_{F}\right)$ as a solution. This leads to a coupled system of equations with $\left(Q_{F}, \underline{J}_{F}, \phi_{F}\right)$ as unknowns:

$$
\begin{array}{r}
\forall \phi_{F}^{\star} \in \mathcal{T}_{F}, \quad \int_{T_{F}} \phi_{F}^{\star}\left(\phi_{F}+\int_{\Omega_{F}}\left(\hat{\alpha}_{F} r^{-1} Q_{F}+\underline{\hat{\beta}}_{F} \cdot H^{-1} \underline{J}_{F}\right) d \Omega\right) d t=0 \\
Q_{F}=\frac{1}{\left\|\phi_{F}\right\|_{T_{F}}^{2}}\left(r \Pi_{F}-\int_{T_{F}} \phi_{F} \hat{\alpha}_{F} d t\right) \quad \text { and } \quad \underline{J}_{F}=\frac{1}{\left\|\phi_{F}\right\|_{T_{F}}^{2}}\left(H \underline{\Xi}_{F}-\int_{T_{F}} \phi_{F} \underline{\beta}_{F} d t\right) \\
\quad \text { with }\left(\Pi_{F}, \underline{\Xi}_{F}\right) \in \mathcal{Z}_{0} \text { such that } \quad \forall\left(\Pi_{F}^{\star}, \Xi_{F}^{\star}\right) \in \mathcal{Z}_{0}, \\
\left.\int_{\Omega_{F}}\left(\Pi_{F} r \Pi_{F}^{\star}+\Xi_{F} \cdot H \underline{\Xi}_{F}^{\star}\right) d \Omega=\int_{\Omega_{F}}\left(\left(\int_{T_{F}} \phi_{F} \hat{\alpha}_{F} d t\right) \Pi_{F}^{\star}+\left(\int_{T_{F}} \phi_{F} \underline{\hat{\beta}}_{F} d t\right) \cdot \underline{\Xi}_{F}^{\star}\right)\right) d \Omega
\end{array}
$$

where $\left\|\phi_{F}\right\|_{T_{F}}^{2}=\int_{T_{F}} \phi_{F}^{2} d t$. System (52) is solved using a fixed-point method between (a) and (b). The number of subiterations is usually small (typically only 1 subiteration will be used in the following examples).

The unknown fields $\left(\Delta p_{F}, \Delta \underline{Z}_{F}\right)$ are approximated by:

$$
\Delta p_{F}(M, t)=P_{F}(M) \pi_{F}(t) \quad \text { and } \quad \Delta \underline{Z}_{F}(M, t)=\underline{\Psi}_{F}(M) \pi_{F}(t)
$$

where $\pi_{F}$ is scalar time function belonging $\mathcal{T}_{F}$, while $\left(P_{F}, \underline{\Psi}_{F}\right)$ is a couple of space fields which belongs to $\mathcal{Z}_{0}$.

In the same manner as for the $S$-physics, one possibility, which is easy to implement, is to chose $P_{F}=\Pi_{F}$ and $\underline{\Psi}_{F}=\Xi_{F}$ and then $\Delta p_{F}=\Pi_{F} \pi_{F}$ and $\Delta \underline{Z}_{F}=\Xi_{F} \pi_{F}$. In Equations (34b), (34c) of the search direction, test functions $q_{F}^{\star}$ and $\underline{W}_{F}^{\star}$ are chosen as $q_{F}^{\star}=r \Pi_{F} \pi_{F}^{\star}$ and $\underline{W}_{F}^{\star}=H \underline{\Xi}_{F} \pi_{F}^{\star}$, with $\pi_{F}^{\star} \in \mathcal{T}_{F}$, which leads to the determination of $\pi_{F}$ by solving:

$$
\forall \pi_{F}^{\star} \in \mathcal{T}_{F}, \quad \int_{T_{F}} \pi_{F}^{\star} \int_{\Omega_{F}}\left(\left(\pi_{F} r \Pi_{F}-\hat{\alpha}_{F}\right) \Pi_{F}+\left(\pi_{F} H \underline{\Xi}_{F}-\underline{\hat{\beta}}_{F}\right) \cdot \Xi_{F}\right) d \Omega d t=0
$$

i.e.:

$$
\forall \pi_{F}^{\star} \in \mathcal{T}_{F}, \quad \int_{\Omega_{F}}\left(r \Pi_{F}^{2}+\Xi_{F} \cdot H \Xi_{F}\right) d \Omega \int_{T_{F}} \pi_{F}^{\star} \pi_{F} d t=\int_{T_{F}} \pi_{F}^{\star} \int_{\Omega_{F}}\left(\hat{\alpha}_{F} \Pi_{F}+\underline{\hat{\beta}}_{F} \cdot \Xi_{F}\right) d \Omega d t
$$




\subsubsection{T-physics}

The thermal problem at decoupled stage is very similar to the fluid problem. Indeed, it is solved with the same procedures, when using the similarity of Table 1.

\begin{tabular}{llllllllll}
\hline$T$-physics & $\theta$ & $R$ & $\underline{X}$ & $\underline{Y}$ & $\mathcal{P}_{T I}$ & $r$ & $H$ & $\hat{\alpha}$ & $\hat{\beta}$ \\
$F$-physics & $p$ & $q$ & $\underline{Z}$ & $\underline{W}$ & $\mathcal{P}_{F I}$ & $u$ & $k / T_{0}$ & $\hat{\gamma}$ & $\underline{\hat{\delta}}$ \\
\hline
\end{tabular}

Table 1: Equivalence of $T$ and $F$ problems at decoupled stage

\subsection{Preliminary stage}

With the previous representation of the solution, we can take the opportunity to reuse the set of already generated space fields, and to only re-compute the time functions, which is less costly: this is the socalled preliminary stage. If the preliminary stage reduces the error indicator with respect to the previous iteration significantly, one can expect that adding a new space function is not mandatory: the decoupled stage is skipped and no new space field is generated. Otherwise, the decoupled stage is applied and a new space field for each fields to be corrected is generated. Full details of this procedure can be found in $[2,3]$.

\subsection{Representation of the unknowns and time-space transfer}

When dealing with the decoupled stage, the algorithm has to transfer time-space fields, at least formerly: indeed, the implementation can avoid the construction and storage of fields depending both on the time step and the space point, by postponing the local stage when the decoupled stage needs a right hand side, and computing it on the fly [42].

In the case where the radial loading approximation is used, the time-space transfer operation (17) can be rewritten. If $\boldsymbol{E}_{1}$ is represented with a product of a discretized space field (with its values in a column vector $V_{1}$ ) by a scalar time function (with its values in a column vector $v_{1}$ ): $\boldsymbol{E}_{1}=V_{1} v_{1}^{T}$, then $\boldsymbol{E}_{2}=\boldsymbol{P}_{21} V_{1} v_{1}^{T} \boldsymbol{p}_{21}^{T}=V_{2} v_{2}^{T}$, where $V_{2}=\boldsymbol{P}_{21} V_{1}$ is the projected column vector corresponding to the space field and $v_{2}=\boldsymbol{p}_{21} v_{1}$ the projected column vector corresponding to the time function.

\subsection{Search direction parameters}

The search direction parameters can be chosen of the form:

$$
\mathbf{L}=t_{S} \mathbf{D}, \quad r=\frac{1}{t_{F} Q} \quad \text { and } \quad u=\frac{C_{d}}{t_{T} T_{0}}
$$

where $t_{S}, t_{F}$ and $t_{T}$ are three arbitrary values, homogeneous to times. It is still important to note that these values do not influence the solution once convergence has been reached but only modify the convergence rate of the algorithm [1].

The choice of these values is made according to the results obtained on the unidimensional problem of [5]. In order to get some information about characteristic times, let us consider the corresponding linear problem (i.e. when neglecting the two nonlinear terms in $(25 \mathrm{e})$ ) written in the unidimensional case. When disregarding the boundary and initial conditions, the admissibility and constitutive relations lead to:

$$
\underbrace{\left[\begin{array}{ccc}
-E & b & 3 \alpha K_{b} \\
b & \frac{1}{Q} & -3 \alpha_{m} \\
3 \alpha K_{b} & -3 \alpha_{m} & \frac{C_{d}}{T_{0}}
\end{array}\right]}_{\boldsymbol{M}} d \frac{d}{d t}\left[\begin{array}{c}
\varepsilon \\
p \\
\theta
\end{array}\right]-\underbrace{\left[\begin{array}{ccc}
0 & 0 & 0 \\
0 & H & 0 \\
0 & 0 & \frac{k}{T_{0}}
\end{array}\right]}_{\boldsymbol{A}} \frac{\partial^{2}}{\partial x^{2}}\left[\begin{array}{c}
\varepsilon \\
p \\
\theta
\end{array}\right]=F(t)
$$

where $F(t)$ is a given right-hand-side term. For the corresponding homogeneous system, if $\lambda_{i}$ and $V_{i}$ are the generalized eigenvalues and eigenvectors of $\boldsymbol{A} V_{i}=\lambda_{i} \boldsymbol{M} V_{i}$, with a regular $\boldsymbol{M}$ (and with $\lambda_{i} \geqslant 0$ ), the previous system leads to decoupled scalar equations: $\dot{y}_{i}-\lambda_{i} \frac{\partial^{2}}{\partial x^{2}} y_{i}=0$ for which the solution is 


\begin{tabular}{|c|c|}
\hline Young modulus of the skeleton & $E=20 \mathrm{GPa}=28.57 \mathrm{M} \mathrm{L}^{-1} \mathrm{~S}^{-2}$ \\
\hline Poisson coefficient of the skeleton & $\nu=0.2$ \\
\hline Bulk modulus of the skeleton & $K_{b}=11.11 \mathrm{GPa}=15.87 \mathrm{M} \mathrm{L}^{-1} \mathrm{~S}^{-2}$ \\
\hline Compressibility of the fluid & $K_{F}=2.15 \mathrm{GPa}=3.07 \mathrm{M} \mathrm{L}^{-1} \mathrm{~S}^{-2}$ \\
\hline Biot modulus & $Q=7.25 \mathrm{GPa}=10.35 \mathrm{M} \mathrm{L}^{-1} \mathrm{~S}^{-2}$ \\
\hline Biot coefficient & $b=0.7$ \\
\hline Porosity & $n=0.2$ \\
\hline Intrinsic permeability of the skeleton & $K=210^{-13} \mathrm{~s} \mathrm{~m}^{2}=210^{-13} \mathrm{~S} \mathrm{~L}^{2}$ \\
\hline Dynamic viscosity of the fluid & $\mu_{F}=0.001 \mathrm{~Pa} \mathrm{~s}=1.4310^{-12} \mathrm{M} \mathrm{L}^{-1} \mathrm{~S}^{-1}$ \\
\hline Permeability of the porous media & $H=210^{-10} \mathrm{~m}^{3} \mathrm{~s} \mathrm{~kg}^{-1}=0.14 \mathrm{~L}^{3} \mathrm{~S} \mathrm{M}^{-1}$ \\
\hline Thermal conductivity of the solid & $k_{S}=85 \mathrm{~W} \mathrm{~m}^{-1} \mathrm{~K}^{-1}=3.5610^{-5} \mathrm{M} \mathrm{L} \mathrm{S}^{-3} \mathrm{~T}^{-1}$ \\
\hline Thermal conductivity of the fluid & $k_{F}=0.61 \mathrm{~W} \mathrm{~m}^{-1} \mathrm{~K}^{-1}=2.5510^{-7} \mathrm{M} \mathrm{L} \mathrm{S}^{-3} \mathrm{~T}^{-1}$ \\
\hline Thermal cond. of the porous media & $k=68 \mathrm{~W} \mathrm{~m}^{-1} \mathrm{~K}^{-1}=2.8510^{-5} \mathrm{M} \mathrm{L} \mathrm{S}^{-3} \mathrm{~T}^{-1}$ \\
\hline Specific mass of the skeleton & $\rho_{S}=1500 \mathrm{~kg} \mathrm{~m}^{-3}=2.1410^{-6} \mathrm{M} \mathrm{L}^{-3}$ \\
\hline Specific mass of the fluid & $\rho_{F}=1000 \mathrm{~kg} \mathrm{~m}^{-3}=1.4310^{-6} \mathrm{M} \mathrm{L}^{-3}$ \\
\hline Specific heat of the solid & $c_{S}=840 \mathrm{~J} \mathrm{~kg}^{-1} \mathrm{~K}^{-1}=2.4610^{5} \mathrm{~L}^{2} \mathrm{~S}^{-2} \mathrm{~T}^{-1}$ \\
\hline Specific heat of the fluid & $c_{F}=4182 \mathrm{~J} \mathrm{~kg}^{-1} \mathrm{~K}^{-1}=1.2310^{6} \mathrm{~L}^{2} \mathrm{~S}^{-2} \mathrm{~T}^{-1}$ \\
\hline Heat capacity of the porous media & $C_{d}=2.110^{6} \mathrm{~J} / \mathrm{K}=0.88 \mathrm{M} \mathrm{L}^{-1} \mathrm{~S}^{-2} \mathrm{~K}^{-1}$ \\
\hline Thermal expansion coef. of the skeleton & $\alpha=810^{-6} \mathrm{~K}^{-1}=2.3410^{-3} \mathrm{~T}^{-1}$ \\
\hline Thermal expansion coef. of the fluid & $\alpha_{F}=2.610^{-4} \mathrm{~K}^{-1}=7.6210^{-2} \mathrm{~T}^{-1}$ \\
\hline Thermal exp. coef. of the porous media & $\alpha_{m}=5.8410^{-5} \mathrm{~K}^{-1}=1.7110^{-2} \mathrm{~T}^{-1}$ \\
\hline Reference temperature & $T_{0}=293 \mathrm{~K}=1 \mathrm{~T}$ \\
\hline
\end{tabular}

Table 2: Material characteristics (porous ceramics)

$[\varepsilon p \theta]^{T}=\sum_{i} \operatorname{diag}\left(y_{i}(x, t)\right) V_{i}$. Each has a physical separated-form solution:

$$
y_{i}=e^{-t / \tau_{i}}\left(A_{i} \cos \frac{x}{\sqrt{\lambda_{i} \tau_{i}}}+B_{i} \sin \frac{x}{\sqrt{\lambda_{i} \tau_{i}}}\right)
$$

To exhibit the characteristic times $\tau_{i}$, boundary conditions are required. If the observation scale is the same for all the physics (the characteristic spatial length $L_{d}$ of the phenomenon that one wishes to capture), the simplest case is to search for internal evolutions of the variables with null boundary conditions for $x=0$ and for $x=L_{d}$. In that case, one gets $A_{i}=0$ and the first non zero root of $\sin \left(L_{d} / \sqrt{\lambda_{i} \tau_{i}}\right)=0$, i.e.

$$
\tau_{i}=\frac{1}{\lambda_{i}}\left(\frac{L_{d}}{\pi}\right)^{2}
$$

For the case of thermo-poroelasticity, one obtains $\lambda_{1}=0$ that corresponds to the solid quantities (since no viscosity is involved, the characteristic time for the solid is related to the external load, and not to the physics). Note that for the thermal-decoupled case $\left(\alpha=\alpha_{m}=0\right)$, the other eigenvalues allow to recover the results of [5]:

$$
\tau_{F}=\frac{1}{H}\left(\frac{1}{Q}+\frac{b^{2}}{E}\right)\left(\frac{L_{d}}{\pi}\right)^{2} \quad \text { and } \quad \tau_{T}=\frac{C_{d}}{k}\left(\frac{L_{d}}{\pi}\right)^{2}
$$

for the fluid and for the thermal problem. In the case of the coupled problem, the analytical expressions are more complex, but do not involve any difficulties.

As an example of material characteristics of the problem that is under consideration, Table 2 reports the parameter values. To avoid bad conditioning of the problem to solve, as for problem (57), a new unit system is used, in order to have a unitary order of magnitude for $E, T_{0}, H$ and $C_{d} / T_{0}$ : lengths are in meters $(\mathrm{L}=\mathrm{m})$, masses in $710^{8} \mathrm{~kg}\left(\mathrm{M}=710^{8} \mathrm{~kg}\right)$, durations in seconds $(\mathrm{S}=\mathrm{s})$ and temperatures in $293 \mathrm{~K}(\mathrm{~T}=293 \mathrm{~K})$. Table 2 also gives the values in this new unit system.

If one considers the linearized problem (57), the corresponding characteristic times are $\tau_{F}=810^{-2} \mathrm{~s}$ and $\tau_{T}=3.1310^{5} \mathrm{~s}$, whereas for the decoupled linearized case, $\tau_{F}=8.210^{-2} \mathrm{~s}$ and $\tau_{T}=3.1210^{5} \mathrm{~s}$, which are very close. The main difficulty here is that the previous thermal characteristic time (computed with conduction phenomena) is very large when compared to the fluid one (with fluid transfer phenomena). Therefore, the previous linearization is not valid, and the heat convectively transported by the fluid is much larger that the heat supplied by diffusion. 
Let us consider again the unidimensional decoupled case $\left(\alpha=\alpha_{m}=0\right)$. We get:

$$
R=\frac{k}{T_{0}} \frac{\partial^{2} \theta}{\partial x^{2}}=\frac{C_{d}}{T_{0}} \dot{\theta}-\frac{\rho_{F} c_{F}}{T_{0}} H \frac{\partial p}{\partial x} \frac{\partial \theta}{\partial x}-\frac{1-3 \alpha_{F} T_{0}}{T_{0}} H\left(\frac{\partial p}{\partial x}\right)^{2}
$$

For a steady-state pore pressure $(\dot{p}=0)$, the pressure gradient is constant. For a unidimensional problem, with a prescribed null pressure on one side, and $P_{d}$ on the other side, it equals $Z=P_{d} / L_{d}$. The temperature evolution is therefore a solution of the advection-diffusion equation:

$$
\frac{\partial^{2} \theta}{\partial x^{2}}=\frac{C_{d}}{k} \dot{\theta}-\frac{\rho_{F} c_{F}}{k} H \frac{P_{d}}{L_{d}} \frac{\partial \theta}{\partial x}-\frac{1-3 \alpha_{F} T_{0}}{k} H\left(\frac{P_{d}}{L_{d}}\right)^{2}
$$

To simplify this expression, we now seek for an adimensional version. With $\theta_{d}$ as a reference temperature difference, the previous expression reads:

$$
\frac{\partial^{2}\left(\theta / \theta_{d}\right)}{\partial\left(x / L_{d}\right)^{2}}+\mathcal{P}_{e}\left[\frac{\partial\left(\theta / \theta_{d}\right)}{\partial\left(x / L_{d}\right)}-\frac{\partial\left(\theta / \theta_{d}\right)}{\partial\left(t / \tilde{\tau}_{T}\right)}\right]=-\mathcal{B}_{r}
$$

where

$$
\mathcal{P}_{e}=\frac{\rho_{F} c_{F}}{k} H P_{d}, \quad \mathcal{B}_{r}=\frac{1-3 \alpha_{F} T_{0}}{k \theta_{d}} H P_{d}^{2} \quad \text { and } \quad \tilde{\tau}_{T}=\frac{\pi^{2}}{\mathcal{P}_{e}} \tau_{T}
$$

$\mathcal{P}_{e}$ is Péclet number; it quantifies the order of magnitude of the heat convectively transported by the fluid compared to the heat supplied by diffusion. $\mathcal{B}_{r}$ is Brinkman number; it quantifies the order of magnitude of the heat source due to the viscous dissipation compared to the heat supplied by conduction. Finally, $\tilde{\tau}_{T}$ is the advection characteristic time.

An analytical solution is

$$
\frac{\theta}{\theta_{d}}=\Theta_{0}\left(\frac{x}{L_{d}}+\frac{t}{\tilde{\tau}_{T}}\right)+\frac{\mathcal{B}_{r}}{\mathcal{P}_{e}} \frac{t}{\tilde{\tau}_{T}}+\Theta_{1}
$$

where $\Theta_{0}$ and $\Theta_{1}$ are constant values. One can check on this expression that the Brinkman number corresponds to a thermal source that tends to increase temperature. This effect can be counterbalanced by thermal advection-diffusion and by thermoelastic effects when the thermal coupling is taken into account (i.e. $\alpha \neq 0, \alpha_{m} \neq 0$ ).

For the problem we are interested in, Table 3 gives the various characteristic quantities: with a reference temperature difference $\theta_{d}$, a characteristic pore pressure $P_{d}$, a characteristic length $L_{d}$, the characteristic times (when assuming a decoupling with the temperature for simplicity of the expressions) are:

- for the pore pressure evolution: $\tau_{F}$,

- for the advection of fluid: $\tau_{W}=\frac{L_{d}}{W}=\frac{L_{d}^{2}}{H P_{d}}$,

- for the heat conductivity: $\tau_{T}$,

- and for the temperature:

$$
\tilde{\tau}_{T}=\frac{\pi^{2}}{\mathcal{P}_{e}} \tau_{T}=\frac{C_{d}}{\rho_{F} c_{F}} \frac{L_{d}^{2}}{H P_{d}}=\frac{C_{d}}{\rho_{F} c_{F}} \tau_{W}
$$

For the search direction parameters, one can selects them according to the results of [5]: the search direction parameter for the $F$-physics is $t_{F}=\tau_{F}$; for the $S$-physics, since no characteristic time is exhibited, $t_{S}$ is chosen to be related to a characteristic time of the loading; in the case of Figure 1, it correspond to $t_{S}=t_{1}$. For the $T$-physics, one may choose $t_{T}=\tilde{\tau}_{T}$, but since this is much larger than the studied time duration $T=0.69 \mathrm{~s}$, the effects to be captured are more related to $t_{T}=T$ which has been chosen here. The chosen values do not influence the converged solution, but may have an impact on convergence rate. There exists an optimal value for them [2], but only the previous rule-of-thumb is used here.

For this particular case, the large value of Péclet number illustrates the fact that this problem is convection-dominated. In such a case, classical finite elements are not the best discretization method, since they may lead to spurious oscillations. Several other possibilities are more suited to deal with such a problem; for instance: finite differences or finite volumes (and discontinuous Galerkin approaches $[43,44])$ with upwinding and flux limiting [45], or stabilized finite elements [46, 47, 48]. Since it is not under the scope of this article, the finite element analysis is nevertheless used herein. Coupling the strategy with different discretizations methods for the different physics is currently under development. 


\begin{tabular}{ll}
\hline reference temperature difference & $\theta_{d}=80 \mathrm{~K}$ \\
characteristic pore pressure & $P_{d}=36 \mathrm{MPa}$ \\
characteristic length & $L_{d}=1.4 \mathrm{~m}$ \\
fluid characteristic times & $\tau_{F}=0.16 \mathrm{~s}$ \\
& $\tau_{W}=270 \mathrm{~s}$ \\
thermic characteristic times & $\tau_{T}=6110 \mathrm{~s}$ \\
& $\tilde{\tau}_{T}=136 \mathrm{~s}$ \\
Péclet number & $\mathcal{P}_{e}=445$ \\
Brinkman number & $\mathcal{B}_{r}=37$ \\
\hline
\end{tabular}

Table 3: Problem characteristics

\subsection{Error indicator and termination criteria}

Unlike classical incremental techniques, the LATIN method produces at each iteration an approximation of the solution over the time-space domain $[0, T] \times \Omega$. Several error indicators can be used to stop the iterations, i.e. to decide when the solution is sufficiently accurate. Since the reference solution $\mathbf{s}_{e x}$ is the intersection of $\mathbf{A}_{\mathbf{d}}$ and $\boldsymbol{\Gamma}$, the distance between $\hat{\mathbf{s}}_{n+1 / 2}$ and $\mathbf{s}_{n}$ is a good error indicator to verify the convergence of the algorithm [49]. A simple measure of this distance is [2]:

$$
\eta=\frac{\left\|\hat{\mathbf{s}}_{n+1 / 2}-\mathbf{s}_{n}\right\|}{\frac{1}{2}\left\|\hat{\mathbf{s}}_{n+1 / 2}+\mathbf{s}_{n}\right\|}
$$

with:

$$
\|\mathbf{s}\|^{2}=\|\varepsilon\|_{\mathbf{D}}^{2}+\|p\|_{Q^{-1}}^{2}+\|\underline{Z}\|_{H}^{2}+\|\theta\|_{k / T_{0}}^{2}+\|\underline{X}\|_{C_{d} / T_{0}}^{2}
$$

where, for the $S$-physics:

$$
\|\varepsilon\|_{\mathbf{D}}^{2}=\int_{T_{S} \times \Omega_{S}}\left(1-\frac{t}{T}\right) \operatorname{Tr}[\dot{\varepsilon} \mathbf{D} \varepsilon] d \Omega d t
$$

for the $F$-physics:

$$
\|p\|_{Q^{-1}}^{2}=\int_{T_{F} \times \Omega_{F}}\left(1-\frac{t}{T}\right) \dot{p} \frac{1}{Q} p d \Omega d t \quad \text { and } \quad\|\underline{Z}\|_{H}^{2}=\int_{T_{T} \times \Omega_{T}}\left(1-\frac{t}{T}\right) \underline{Z} \cdot H \underline{Z} d \Omega d t
$$

and for the $T$-physics:

$$
\|\theta\|_{k / T_{0}}^{2}=\int_{T_{T} \times \Omega_{T}}\left(1-\frac{t}{T}\right) \dot{\theta} \frac{k}{T_{0}} \theta d \Omega d t \quad \text { and } \quad\|\underline{X}\|_{C_{d} / T_{0}}^{2}=\int_{T_{T} \times \Omega_{T}}\left(1-\frac{t}{T}\right) \underline{X} \cdot \frac{C_{d}}{T_{0}} \underline{X} d \Omega d t
$$

Previous quantities have been defined in the continuous case and their computation obviously implies some transfers when dealing with the time-space discretized fields.

\section{NUMERICAL RESULTS}

All of the 2D resolution has been implemented into the Matlab ${ }^{\circledR}$ environment and interfaced with the finite element code Cast3M ${ }^{\mathrm{TM}}[50,51]$ (CEA Saclay, France) and gmsh ${ }^{\mathrm{TM}}[52,53]$ for pre and posttreatment facilities. The example concerns the filter, made of porous material, already described in subsection 1.2 and Table 2.

The example concerns again the pressurized filter made of porous ceramics of Section 1.2. The inflow pressure $p_{d}$ and the temperature $\theta_{d}$ are prescribed for the $F$-physics and for the $T$-physics in the round bores, while null pressure and temperature are prescribed on the external boundary. The pressure $p_{d}$ leads to boundary conditions on the $S$-physics: a prescribed force $\underline{F}_{d}=-p_{d} \underline{n}$ on the same part of the boundary. The maximum prescribed pressure is $p_{1}=P_{d}$ at the time $t_{1}=0.34 \mathrm{~s}$, while the maximum temperature is $\theta_{1}=\theta_{d}$, Figure 6 . The initial conditions are null temperature and pore pressure.

\subsection{Case of identical discretizations}

The first results are obtained with the LATIN method, but with a unique finite element discretization: the mesh of the whole filter section is depicted on Figure 7 (the simulation is performed on a quarter 

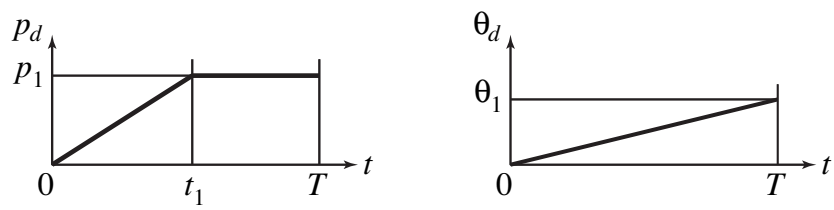

Figure 6: Prescribed pressure $p_{d}$ and temperature $\theta_{d}$ in the round bores

of it), with P2 elements (6-node triangles) for the displacement of the $S$-physics domain and P1 linear interpolation (also continuous throughout the elements) for the pore pressure of the $F$-physics and for the temperature of the $T$-physics $[54,55]$. When a unique mesh is used, the local stage is performed at each integration point of each element (here, the Gauss points of the isoparametric P2 element). The time discretization is the same for all the physics: $T_{S}=T_{F}=T_{T}$; the studied time duration is $T=0.69 \mathrm{~s}$, discretized in $n_{T}=120$ regular steps, and the integration scheme is the time discontinuous Galerkin method with linear interpolation on each time slab.

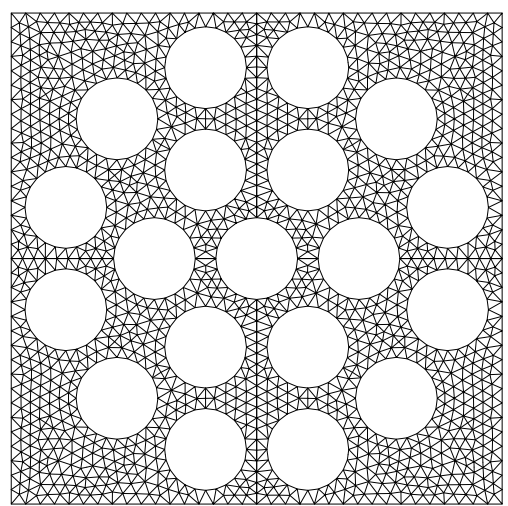

Figure 7: Quasi-uniform mesh of the domain

The convergence of the algorithm is shown on Figure 8, which reports the evolution of the error indicator $\eta$ versus the iteration number. The convergence is estimated to be reached after 40 iterations, when the error indicator is less than $2 \%$. Figure 9 plots the obtained solution at the end of the studied time interval.

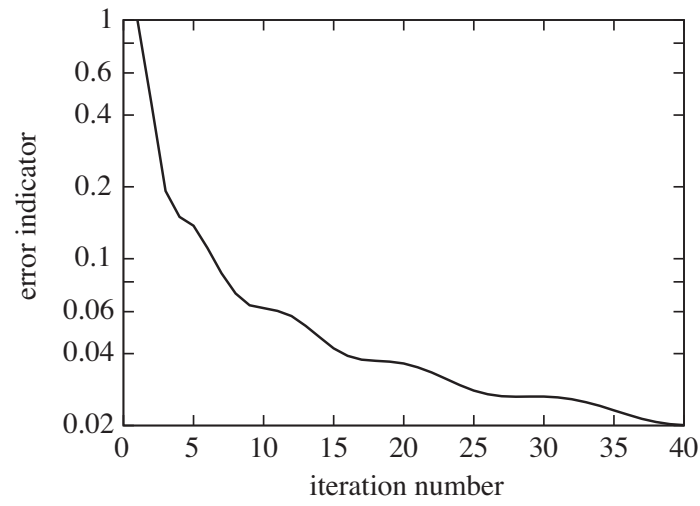

Figure 8: Evolution of the error indicator $\eta$ along the iterations

To illustrate the behavior of the algorithm, Figure 10 reports the time evolution of the maximum norm (over the domain) of several fields. Each graph plots the admissible field produced by the decoupled stage and the field produced by the local stage at the initialization (iteration 1) and at half-way of the convergence (iteration 20). As the solution is improved along the iterations, the previous two fields tend to superpose to each other. 


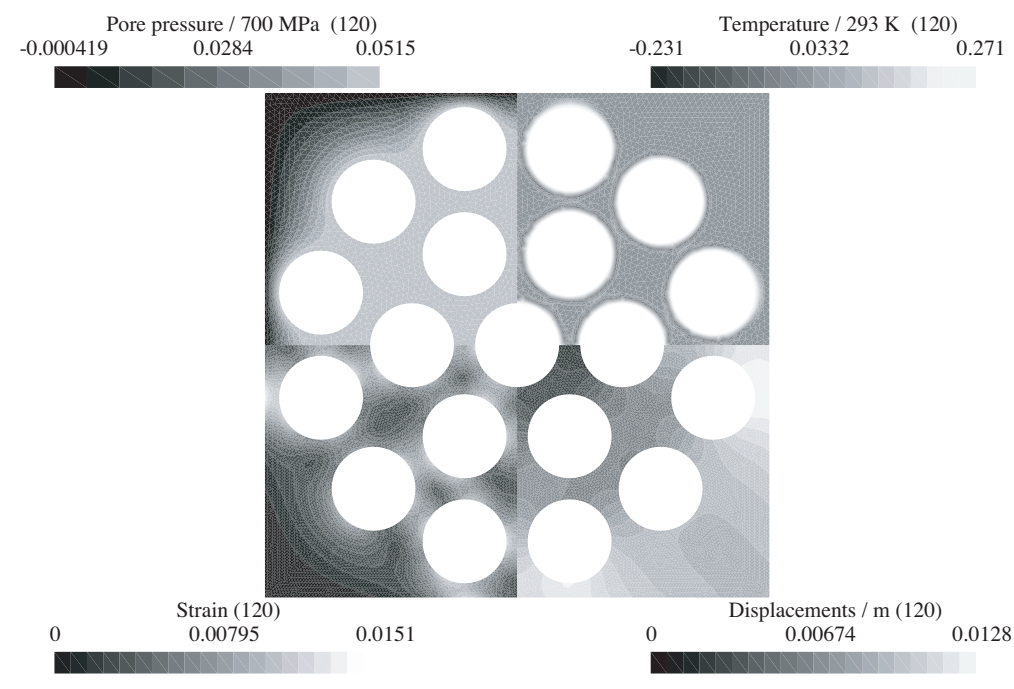

Figure 9: Solution at $t=T$ : pore pressure, temperature, maximum principal strain and norm of the displacement
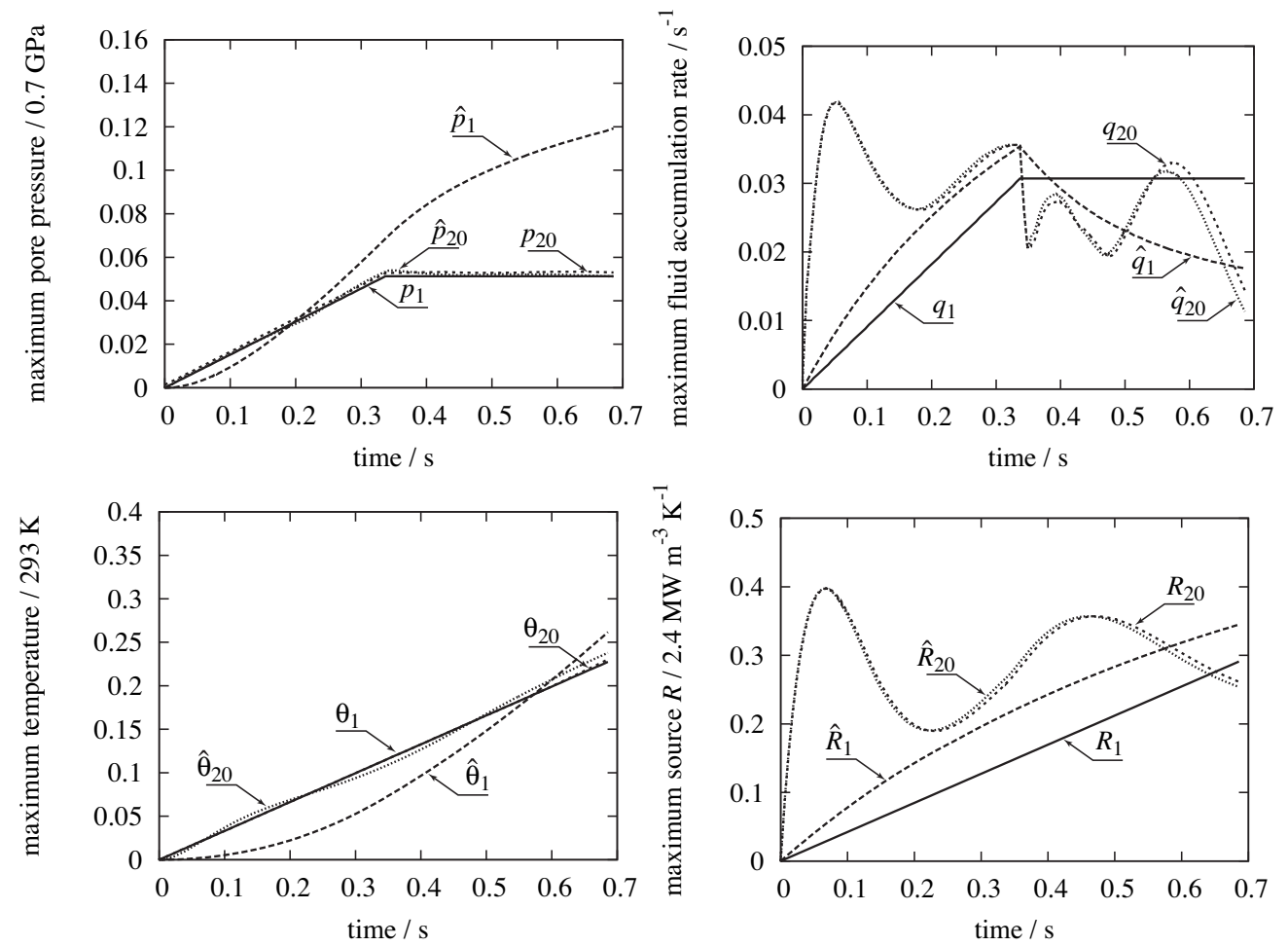

Figure 10: Evolution of the norm of fields $(p, \hat{p}),(q, \hat{q}),(\theta, \hat{\theta})$ and $(R, \hat{R})$ at iterations 1 and 20

\subsection{Case of different space-time discretizations}

With the previous space-time transfer operators, one can use different discretizations for each physics. Here, to exemplify the feasibility of the approach, we select a priori discretizations for both the space and the time aspects. For the space aspect, Figure 11 shows the used discretizations (P2 elements for the displacement of the $S$-physics, P1 elements for the other physics, and for the interface). Concerning the time discretizations, we chose 70 time steps for the $S$-physics, 120 time steps for the $F$-physics, 45 time steps for the $T$-physics, and 60 time steps for the interface time discretization. Figure 12 shows the evolution of the chosen time steps lengths for each physics and for the interface.

The same error indicator can be used, once the admissible fields are projected back to the interface: the error indicator is therefore computed on the interface space-time discretization. Nevertheless, due to the fact that the discretization spaces are different on the interface and on the physical domains, the 


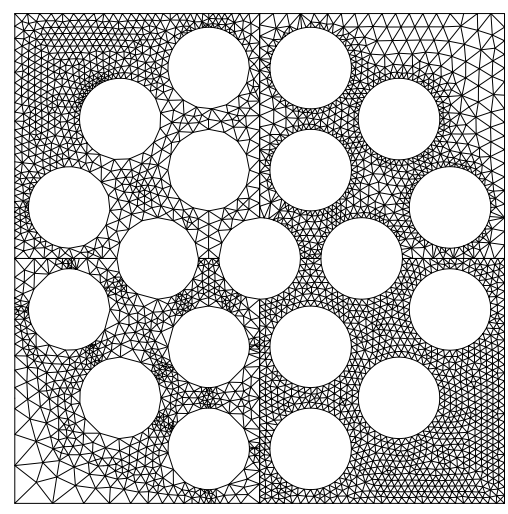

Figure 11: Incompatible meshes of the domain: mesh of the $F$-physics (left, top), $T$-physics (right, top), $S$-physics (left, bottom) and of the interface (right, bottom)

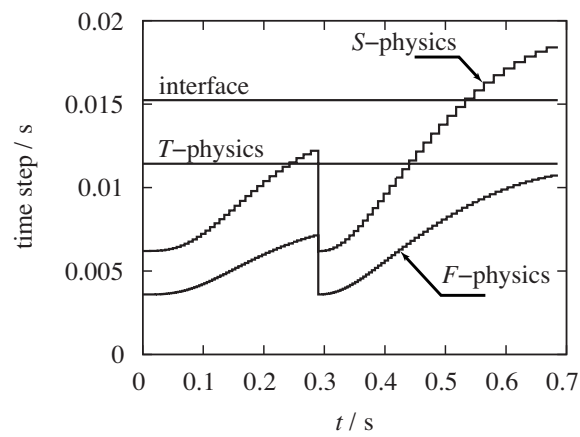

Figure 12: Evolutions of time step lengths for each physics and for the interface

previous indicator does not decrease up to zero. The convergence is declared when this indicator stops to decrease and stagnates. This residual level is characteristic of the discretization errors. Figure 13 shows the solution at the end of the studied time interval, when the convergence is reached (after 40 iterations in that example).

To compare the results with the ones obtained in the case of identical discretizations, Figure 14 reports the evolution of the same quantities as in Figure 10. It has to be noted that, when different discretizations are used, the admissible fields produced by the decoupled and the fields produced by the local stage are not defined any more on the same mesh and then do not necessary tend to superpose to each other along the iterations (see for example the case of $q$ and $\hat{q}$ in Figure 14). For the same reason, the solutions which are generated using identical or different discretizations can also be different.

The good agreement between the results presented in Figure 9 and in Figure 13 validates the possibility of dealing with different (and also incompatible) time-space discretizations.

\section{CONCLUSIONS}

In this paper, we proposed a partitioned strategy suitable for the simulation of coupled multiphysics problems, which is based on the LATIN method. The main issue of this work concerned the treatment of the different scales which usually arise with the different physics and especially the coupling of different space and time discretizations. The feasibility has been shown on a $2 \mathrm{D}$ nonlinear thermo-poroelastic problem.

The basic idea was the introduction of an 'interface between the physics' which had its own space and time discretizations. This interface, on which all the couplings are satisfied, allows to deal with a multiphysics problem with a minimum effort of implementation and offers a convenient framework for the use of different time or space discretizations due to its modularity.

In the proposed example, the time and space discretizations where defined a priori; the automatic determination of the optimum discretizations for the different physics, and for the interface, would be a further step on the proposed computational strategy. Using the projectors defined in this article, an adaptation of the discretizations along the iterations is directly conceivable. 


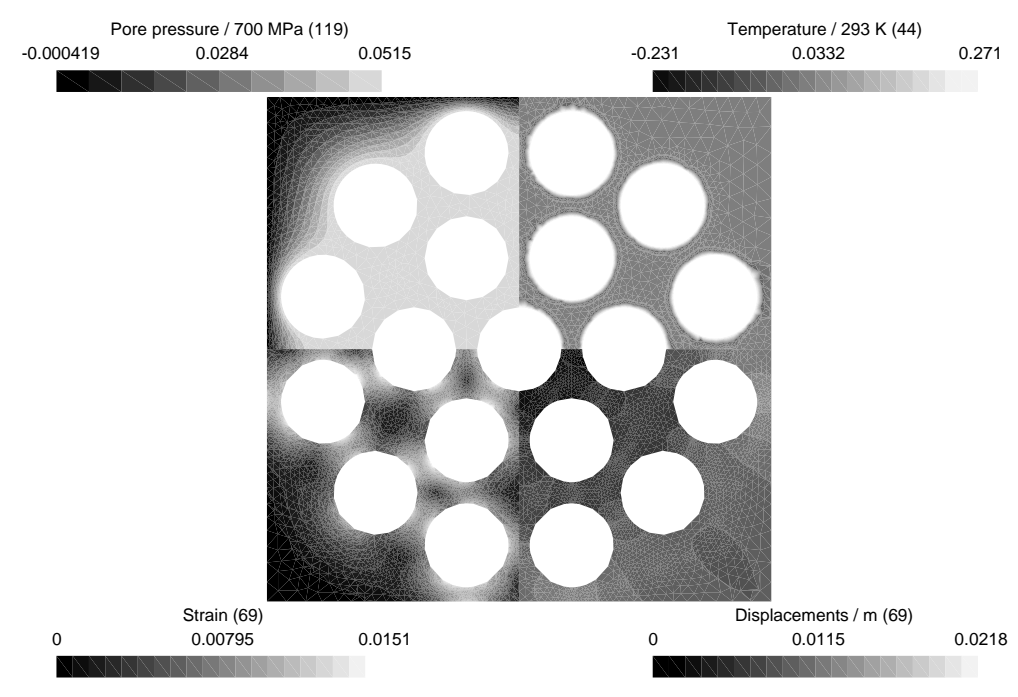

Figure 13: Solution at $t=T$ : pore pressure, temperature, maximum principal strain and norm of the displacement
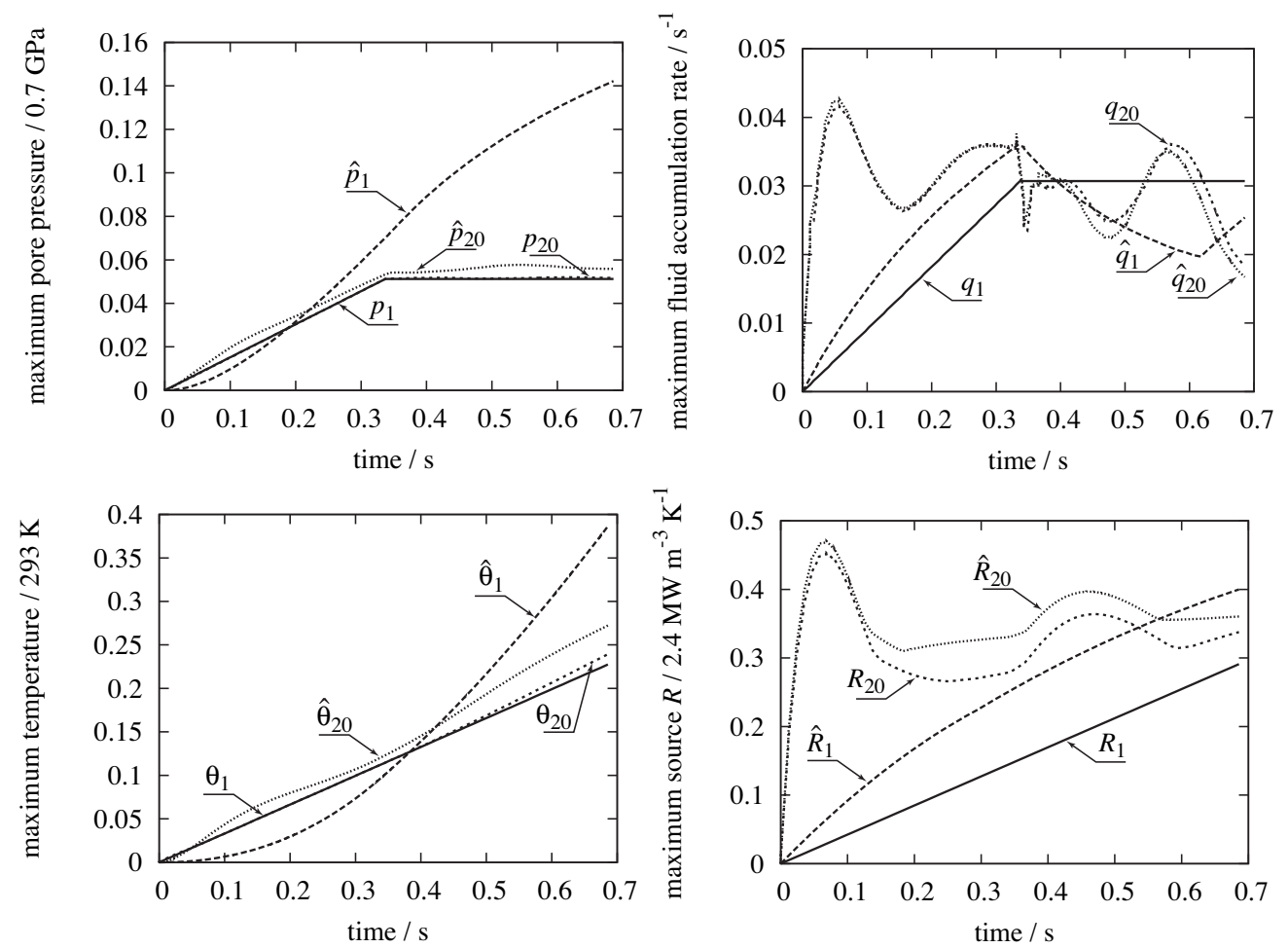

Figure 14: Evolution of the norm of fields $(p, \hat{p}),(q, \hat{q}),(\theta, \hat{\theta})$ and $(R, \hat{R})$ at iterations 1 and 20

A second major difficulty in the treatment of multiphysics problems is the significant cost in terms of storage and data exchange. Further works are in progress to propose a general time-space approximation framework, which is expected to result in a significant decrease of the computational and storage costs.

\section{References}

[1] P. Ladevèze. Nonlinear computational structural mechanics - New approaches and non-incremental methods of calculation. Springer Verlag, 1999.

[2] D. Dureisseix, P. Ladevèze, and B. A. Schrefler. A computational strategy for multiphysics problems - application to poroelasticity. International Journal for Numerical Methods in Engineering, 56(10):1489-1510, 2003. 
[3] D. Dureisseix, P. Ladevèze, D. Néron, and B. A. Schrefler. A multi-time-scale strategy for multiphysics problems: application to poroelasticity. International Journal for Multiscale Computational Engineering, 1(4):387-400, 2003.

[4] P. Ladevèze, D. Néron, and B. A. Schrefler. A computational strategy suitable for multiphysics problems. In Proceedings of the International Conference on Computational Methods for Coupled Problems in Science and Engineering, Santorini Island, Greece, 2005.

[5] D. Néron and D. Dureisseix. A computational strategy for poroelastic problems with a time interface between coupled physics. International Journal for Numerical Methods in Engineering, 2007. to appear.

[6] F. J. Blom. A monolithic fluid-structure interaction algorithm applied to the piston problem. Computer Methods in Applied Mechanics and Engineering, 167:369-391, 1998.

[7] P. Gosselet, V. Chiaruttini, Ch. Rey, and F. Feyel. A monolithic strategy based on an hybrid domain decomposition method for multiphysic problems. application to poroelasticity. Revue Européenne des Éléments Finis, 13(5/7):523-534, 2004.

[8] C. Michler, S. J. Hulshoff, E. H. van Brummelen, and R. de Borst. A monolithic approach to fluid-structure interaction. Computers and Structures, 33:839-848, 2004.

[9] Ch. Farhat, M. Lesoinne, and P. LeTallec. Load and motion transfer algorithms for fluid/structure interaction problems with non-matching discrete interfaces: momentum and energy conservation. optimal discretisation and application to aeroelasticity. Computer Methods in Applied Mechanics and Engineering, 157:95-114, 1998.

[10] Ch. Farhat, K. G. van der Zee, and Ph. Geuzaine. Provably second-order time-accurate looselycoupled solution algorithms for transient nonlinear computational aeroelasticity. Computer Methods in Applied Mechanics and Engineering, 195:1973-2001, 2006.

[11] P. Causin, J.-F. Gerbeau, and F. Nobile. Added-mass effect in the design of partitioned algorithms for fluid-structure problems. Computer Methods in Applied Mechanics and Engineering, 194(4244):4506-4527, 2005.

[12] H. G. Matthies and J. Steindorf. Partitioned but strongly coupled iteration schemes for nonlinear fluid-structure interaction. Computers and Structures, 80:1991-1999, 2002.

[13] O. Coussy. Poromechanics. John Wiley \& Sons, 2004.

[14] R. W. Lewis and B. A. Schrefler. The finite element method in the static and dynamic deformation and consolidation of porous media. John Wiley \& Sons, 2nd edition, 1998.

[15] O. C. Zienkiewicz and R. L. Taylor. The finite element method. Mc Graw-Hill, fourth edition, 1991.

[16] P. Ladevèze, O. Loiseau, and D. Dureisseix. A micro-macro and parallel computational strategy for highly heterogeneous structures. International Journal for Numerical Methods in Engineering, 52(1-2):121-138, 2001.

[17] P. Ladevèze and A. Nouy. A multiscale computational method with time and space homogenization. Comptes-Rendus de l'Académie des Sciences, IIb(330):683-689, 2002.

[18] P. Ladevèze and A. Nouy. On a multiscale computational strategy with time and space homogenization for structural mechanics. Computer Methods in Applied Mechanics and Engineering, 192:30613087, 2003.

[19] D. Dureisseix and H. Bavestrello. Information transfer between incompatible finite element meshes: application to coupled thermo-viscoelasticity. Computer Methods in Applied Mechanics and Engineering, 195(44-47):6523-6541, 2006.

[20] J. O'Rourke. Computational geometry in C. Cambridge University Press, second edition, 1998.

[21] C. Bernardi, Y. Maday, and A. T. Patera. A new nonconforming approach to domain decomposition: the mortar element method. In H. Brezis and J.-L. Lions, editors, Nonlinear partial differential equations and their applications, pages 13-51. Collège de France seminar, Pitman, Paris, 1994. 
[22] F. Ben Belgacem and Y. Maday. The mortar finite element method for three dimensional finite elements. Mathematical Modelling and Numerical Analysis, 31:289-302, 1997.

[23] K. Eriksson, C. Johnson, and V. Thomée. Time discretization of parabolic problems by the discontinuous galerkin formulation. RAIRO Modélisation Mathématique et Analyse Numérique, 19:611-643, 1985.

[24] M. Borri and C. Bottaso. A general framework for interpreting time finite element formulations. Computational Mechanics, (13):133-142, 1993.

[25] X. Li, Z. Liu, and R. W. Lewis. Mixed finite element method for coupled thermo-hydro-mechanical process in poro-elasto-plastic media at large strains. International Journal for Numerical Methods in Engineering, 64:667-708, 2005.

[26] Ch. Rey and V. Chiaruttini. An efficient multiscale parallel-in-time strategy for nonlinear poroplastic problems. In Proceedings of the 8th ESAFORM Conference on Material Forming, pages 137-140, 2005.

[27] J.-L. Lions, Y. Maday, and G. Turinici. Résolution d'EDP par un schéma en temps "pararéel". Comptes-Rendus de l'Académie des Sciences, I(332):661-668, 2001. In french.

[28] Ch. Farhat and M. Chandesris. Time-decomposed parallel time-integrators: theory and feasibility studies for fluid, structure, and fluid-structure applications. International Journal for Numerical Methods in Engineering, 58:1397-1434, 2003.

[29] C. A. Felippa and K. C. Park. Staggered transient analysis procedures for coupled mechanical systems: formulation. Computer Methods in Applied Mechanics and Engineering, 24:61-111, 1980.

[30] C. A. Felippa and T. L. Geers. Partitioned analysis for coupled mechanical systems. Engineering Computation, 5:123-133, 1988.

[31] R. W. Lewis, B. A. Schrefler, and L. Simoni. Coupling versus uncoupling in soil consolidation. International Journal for Numerical and Analytical Methods in Geomechanics, 15:533-548, 1991.

[32] J.-P. Morand and R. Ohayon. Fluid-structure interaction: applied numerical methods. John Wiley \& Sons, 1995.

[33] S. Piperno, C. Farhat, and B. Larrouturou. Partitioned procedures for the transient solution of coupled aeroelastic problems. Part I: model problem, theory and two-dimensional application. Computer Methods in Applied Mechanics and Engineering, 124:79-112, 1995.

[34] C. Farhat and M. Lesoinne. Two efficient staggered algorithms for the serial and parallel solution of three-dimensional nonlinear transient aeroelastic problems. Computer Methods in Applied Mechanics and Engineering, 182:499-515, 2000.

[35] N. Maman and C. Farhat. Matching fluid and structure meshes for aeroelastic computations: a parallel approach. Computers and Structures, 54(4):779-785, 1995.

[36] C. Farhat, M. Lesoinne, and P. LeTallec. Load and motion transfer algorithms for fluid/structure interaction problems with non-matching discrete interfaces: Momentum and energy conservation, optimal discretization and application to aeroelasticity. Computer Methods in Applied Mechanics and Engineering, 157:95-114, 1998.

[37] A. Beckert. Coupling fluid (CFD) and structural (FE) models using finite interpolation elements. Aerospace Science and Technology, 47:13-22, 2000.

[38] C. A. Felippa, K. C. Park, and C. Farhat. Partitioned analysis of coupled mechanical systems. Computer Methods in Applied Mechanics and Engineering, 190:3247-3270, 2001.

[39] H. G. Matthies and J. Steindorf. Partitioned strong coupling algorithms for fluid-structure interaction. Computers and Structures, 81:805-812, 2003.

[40] T. I. Zohdi. Modeling and simulation of a class of coupled modeling and simulation of a class of coupled thermo-chemo-mechanical processes in multiphase solids. Computer Methods in Applied Mechanics and Engineering, 193:679-699, 2004. 
[41] D. Néron, P. Ladevèze, D. Dureisseix, and B. A. Schrefler. Accounting for nonlinear aspects in multiphysics problems: application to poroelasticity. Lecture Notes in Computer Science, 3039(IV):612$620,2004$.

[42] J.-Y. Cognard. Simulation sur ordinateurs à architecture parallèle de structures viscoplastiques sous chargements cycliques. Revue Européenne des Éléments Finis, 5(1):101-119, 1996. In french.

[43] B. Cockburn and C. W. Shu. The local discontinuous Galerkin method for time dependent convection-diffusion systems. SIAM Journal of Numerical Analysis, 35:2440-2463, 1998.

[44] C. Dawson and J. Proft. Coupling of continuous and discontinuous galerkin methods for transport problems. Computer Methods in Applied Mechanics and Engineering, 191:3213-3231, 2002.

[45] R. LeVeque. Finite Volume Methods for Hyperbolic Problems. Cambridge University Press, Cambridge, 2002.

[46] A. N. Brooks and T. J. R. Hughes. Streamline upwind Petrov-Galerkin formulations for convection dominated flows with particular emphasis on the incompressible Navier-Stokes equations. Computer Methods in Applied Mechanics and Engineering, 32:199-259, 1982.

[47] E. Oñate and M. Manzán. A general procedure for deriving stabilized space-time finite element methods for advective-diffusive problems. International Journal for Numerical Methods in Fluids, 31(1):203-221, 1999.

[48] T. J. R. Hughes, G. Scovazzi, and L. P. Franca. Multiscale and stabilized methods. In E. Stein, R. de Borst, and T. J. R. Hughes, editors, Encyclopedia of Computational Mechanics, volume 3, chapter 2. John Wiley \& Sons, Ltd., 2004.

[49] P. Ladevèze and J.-P. Pelle. Mastering calculations in linear and nonlinear mechanics. Springer, 2004.

[50] P. Verpeaux, T. Charras, and A. Millard. CASTEM 2000 : une approche moderne du calcul des structures. In J.-M. Fouet, P. Ladevèze, and R. Ohayon, editors, Calcul des Structures et Intelligence Artificielle, volume 2, pages 261-271. Pluralis, Paris, France, 1988. http://www-cast3m.cea.fr/.

[51] P. Verpeaux, A. Millard, T. Charras, and A. Combescure. A modern approach of large computer codes for structural analysis. In 10th International Conference on Structural Mechanics in Reactor Technology, Los Angeles, August 1989.

[52] Ch. Geuzaine and J.-F. Remacle. Gmsh: a finite element mesh generator with built-in pre- and post-processing facilities. Available from: http://www.geuz.org/gmsh/.

[53] J.-F. Remacle, N. Chevaugeon, E. Marchandise, and Ch. Geuzaine. Efficient visualization of highorder finite elements. International Journal for Numerical Methods in Engineering, 69(4):750-771, 2007.

[54] O. C. Zienkiewicz, S. Qu, R. L. Taylor, and S. Nakazawa. The patch test for mixed formulations. International Journal for Numerical Methods in Engineering, 23:1873-1883, 1986.

[55] F. Brezzi and M. Fortin. Mixed and hybrid finite element methods, volume 15 of Computational Mathematics. Springer, 1991. 